\title{
Insensitivity of the complexity rate of change to the conformal anomaly and Lloyd's bound as a possible renormalization condition
}

\author{
Daniel Ávila@, César Díaz@ ${ }^{\dagger}$ and Leonardo Patiño®* \\ Departamento de Física, Facultad de Ciencias, Universidad Nacional Autónoma de México, \\ Apartado Postal 70-542, Ciudad de México 04510, México \\ Yaithd D. Olivas $\oplus^{\S}$ \\ Departamento de Física de Altas Energias, Instituto de Ciencias Nucleares, \\ Universidad Nacional Autónoma de México, Apartado Postal 70-543, México, \\ Ciudad de México 04510, México
}

(Received 1 July 2021; accepted 10 August 2021; published 9 September 2021)

\begin{abstract}
We determine the effect on the computational complexity of a conformal anomaly using the complexity $=$ action prescription of the gauge/gravity correspondence. To allow the involvement of said anomaly, we extend previous studies to include arbitrary values for the anisotropic parameter and the magnetic field, respectively, on the Mateos-Trancanelli and D'Hoker-Kraus holographic models. Our main result is that the rate of change of the computational complexity is independent of the conformal anomaly in both cases. In addition, this allows us to show that, if so desired, the saturation of Lloyd's bound at infinite time can be used as a renormalization condition.
\end{abstract}

DOI: 10.1103/PhysRevD.104.066011

\section{INTRODUCTION}

In recent years, the AdS/CFT correspondence [1] has served as a bridge that connects concepts of quantum information theory and gravity. The holographic dictionary $[2,3]$ typically relates bulk and boundary physical quantities, such as the entanglement entropy, whose holographic dual is the area of an extremal surface in the bulk of the spacetime [4,5], and the entanglement of purification, which is dual to the area of the minimal cross section of the entanglement wedge [6]. However, there is now evidence that the black hole interior also encodes important information about the dual gauge theory, concerning not only information theory [7-9] but other systems as well, such as superconductors [10]. An important step in this direction was the conjecture, based on studies of the time evolution of the entanglement entropy [11], that the computational complexity of the boundary state is also affected by the interior of the black hole.

There are two main proposals for this connectionnamely, the complexity $=$ volume $(\mathrm{CV})[12,13]$ and the

\footnotetext{
*davhdz06@ciencias.unam.mx

†cadh@ciencias.unam.mx

teopj@ciencias.unam.mx

§yaithd.olivas@correo.nucleares.unam.mx
}

Published by the American Physical Society under the terms of the Creative Commons Attribution 4.0 International license. Further distribution of this work must maintain attribution to the author(s) and the published article's title, journal citation, and DOI. Funded by SCOAP. complexity $=$ action $(\mathrm{CA})[14,15]$ conjectures. In the CV conjecture, the complexity is dual to the volume of a certain extremal region in the bulk [16]. However, the precise prescription includes some unsatisfactory features like the introduction of an arbitrary scale and the necessity for a special foliation of spacetime. While these issues were tackled, the CA conjecture was proposed as a refinement in which complexity is dual to the action of the gravitational theory evaluated in a region known as the Wheeler-DeWitt (WDW) patch $[14,15]$, with the precise relation being

$$
C=\frac{S_{W D W}}{\pi} .
$$

The WDW patch is the region enclosed by past and future light sheets sent into the bulk from a constant time slice on the boundary, where the complexity is meant to be evaluated. In other words, the WDW patch is the domain of dependence of any Cauchy surface in the bulk which asymptotically approaches said time slice at the boundary.

The gravity setup considered in $[14,15]$ was a two-sided black hole geometry. From the point of view of the gauge theory, this is dual to a thermofield double (TFD) state which, denoting the two asymptotic regions as the left $(L)$ and right $(R)$ boundaries, can be written schematically as

$$
|T F D\rangle=\frac{1}{Z^{\frac{1}{2}}} \sum_{n} e^{-\frac{\beta E_{n}}{2}} e^{-E_{n}\left(t_{L}+t_{R}\right)}\left|E_{n}\right\rangle_{L}\left|E_{n}\right\rangle_{R},
$$

where $\beta$ is the inverse of the temperature. It is important to note that the TFD state is invariant under time evolution 
with the Hamiltonian $H=H_{L}-H_{R}$, which means that the state is invariant under the transformations $t_{L} \rightarrow t_{L}+\Delta t$ and $t_{R} \rightarrow t_{R}-\Delta t$. This implies that the TFD state depends only on time through the combination $\tau=t_{L}+t_{R}$.

The late-time behavior of the rate of change of the complexity of certain black holes was studied in $[14,15]$ using both the CV and CA conjectures. In the case of a planar five-dimensional anti-de Sitter (AdS)Schwarzschild black hole, the CV late-time calculation leads to

$$
\lim _{t \rightarrow \infty} \frac{d C}{d \tau}=4 \pi E,
$$

while in the case of CA the behavior is

$$
\lim _{t \rightarrow \infty} \frac{d C}{d \tau}=\frac{2 E}{\pi},
$$

which is consistent with Lloyd's bound [17] on the rate of computation by a system with energy $E$,

$$
\frac{d C}{d \tau} \leq \frac{2 E}{\pi} .
$$

It was later shown [18] that the correct evaluation of the gravitational action over the WDW patch requires the addition of boundary terms $S_{\text {surf }}$ resulting from the codimension-1 null hypersurfaces that delimit part of it, and of joint terms $S_{\text {joint }}$ arising from the codimension- 2 intersections of the light sheets with the boundary and the singularity. It was also observed that there is an ambiguity in $S_{\text {surf }}$ coming from the normalization of normal vectors to the null segments of the boundary, and that it is necessary to add a counterterm $S_{\text {null }}$ in order to remove it. Hence, the full action can be written schematically as

$$
S=S_{\text {bulk }}+S_{\text {surf }}+S_{\text {joint }}+S_{\text {null }} .
$$

The complete action (6) made it possible in [19] to study the behavior at all times for the rate of change of the complexity. It was shown that, for the black holes that they consider, the CV prescription saturates Lloyd's bound (5) from below at late times, while the CA prescription saturates it from above. In other words, it was shown that the CA prescription violates Lloyd's bound for any finite time, saturating it only at infinite time. This led to question the validity of Lloyd's bound in the holographic context, whether it is reasonable to expect it to hold, and under which conditions it would hold [20]. For instance, it was found in [21] that Lloyd's bound is violated for nonconmutative supersymmetric Yang-Mills theories, and the same was shown in $[22,23]$ for the case of Lifshitz and hyperscaling violating theories, even at late times. Other examples of violations of Lloyd's bound can be found in [24-27]. There is also the question of exactly what kind of complexity is dual to the holographic complexity [28]. For example, Nielsen's circuit complexity [29] for a charged thermofield double state was compared to the holographic result using CA in [30], with different saturation times for Lloyd's bound obtained depending on the approach.

Recently, the effect that a spatial anisotropy in the gauge theory can have on the rate of change of the complexity was studied in [31] using certain limits of the Cheng-Ge-Sin model, the Mateos-Trancanelli (MT) anisotropic model [32,33], and the D'Hoker-Krauss (DK) magnetic model [34]. The results show that at late times Lloyd's bound is saturated from above for small anisotropies in the case of the MT model and for an infinite magnetic field intensity in the case of the DK model. However, both models feature a conformal anomaly for any nonvanishing $a$ in the case of the MT model and any nonvanishing $b$ in the case of the DK model. The conformal anomaly (also known as the Weyl or scale anomaly) was first studied in the holographic context in [35], where it was shown that the Lagrangian of the theory is no longer invariant under rescalings of the holographic radial coordinate when a logarithmic divergence appears in the holographic renormalization procedure [36-39]. It was shown in [40] that this is related to the appearance of a reference energy scale that is independent of those in the classic counterpart. The existence of this parameter that is not present in the classical theories manifests itself by making some physical observables, such as the expectation value of the stressenergy tensor of the gauge theory, to have anomalous behavior under scale transformations. The nontrivial manner in which the conformal anomaly present $[35-37,40]$ in both the MT and DK models can affect the physics of the theory was not studied as part of the limits above, as it becomes a subdominant contribution of order $(a / T)^{4}$ $[32,33]$ for the first of these models, while the second undergoes a dimensional reduction [41] in the $b / T^{2} \rightarrow \infty$ limit that renders the four-dimensional conformal anomaly inapplicable.

The main objective of this work is to determine the effect that the conformal anomaly has on the complexity of the state, and in particular whether or not it has an impact on Lloyd's bound. In order to achieve this, we extend the study presented in [31] to include arbitrary anisotropies and magnetic field intensities, finding as our main result that, even if the complexity itself is affected by the conformal anomaly, its rate of change is not, making it independent of the renormalization scale. Given that the energy of the state depends on this scale, we also show that the saturation of Lloyd's bound at infinite time can be used, if so desired, as a renormalization condition and use a scheme related constant that appears in the construction to preserve it at all values of $a / T$ in the MT model and of $b / T^{2}$ for the DK case.

The manuscript is organized as follows. In Sec. II we review the construction of both the MT and DK models, 
explaining the role of the conformal anomaly in both cases. Details about the construction of the interior solutions can be found in the Appendix A. In Sec. III we explain the construction of the WDW patch for both models, and in Sec. IV we compute the complexity of the TFD state, along with its rate of change, using the CA prescription. We close by discussing our results in Sec. V.

\section{GRAVITY SETUP}

\section{A. Anisotropic black branes}

The MT anisotropic model [32,33] is a ten-dimensional family of solutions to type IIB supergravity (SUGRA). However, for our purposes it suffices to consider its reduction to five dimensions, whose action in the Einstein frame is given by [42]

$$
\begin{aligned}
S= & \frac{1}{16 \pi G_{5}} \int d x^{5} \sqrt{-g}\left(R+\frac{12}{L^{2}}-\frac{1}{2}(\partial \phi)^{2}-\frac{1}{2} e^{2 \phi}(\partial \chi)^{2}\right) \\
& +\frac{1}{16 \pi G_{5}} \int d x^{4} \sqrt{-\gamma} 2 K,
\end{aligned}
$$

where $\phi, \chi$, and $g_{\mu \nu}$ are the dilaton, the axion, and metric fields, respectively. $G_{5}$ is the five-dimensional Newton constant and $L$ is the $\mathrm{AdS}_{5}$ radius, which we will set to unity without loss of generality [43]. The second integral is the York-Gibbons-Hawking (YGH) surface term, in which $\gamma_{i j}$ is the induced metric at the boundary and $K$ its extrinsic curvature.

Every member of the MT family of solutions is part of the Ansatz

$$
\begin{aligned}
d s^{2}= & e^{\frac{-\phi}{2}} r^{2}\left(\frac{d r^{2}}{r^{4} \mathcal{F}(r)}-\mathcal{F}(r) \mathcal{B}(r) d t^{2}\right) \\
& +e^{\frac{-\phi}{2}} r^{2}\left(d x^{2}+d y^{2}+e^{-\phi} d z^{2}\right), \\
\chi= & a z, \quad \phi=\phi(r) .
\end{aligned}
$$

Here $r$ is the $\mathrm{AdS}_{5}$ radial coordinate, in terms of which the boundary is located at $r \rightarrow \infty$. All these backgrounds feature a horizon located at $r=r_{h}$, where the metric function $\mathcal{F}(r)$ vanishes. The parameter $a$ measures the degree of anisotropy in the geometry and its relation to a density of D7-branes that are smeared in the geometry from the ten-dimensional perspective (see [32,33] for more details). Meanwhile, the temperature of each member of the family is given by

$$
T=\frac{e^{-\frac{1}{2} \phi\left(r_{h}\right)} \sqrt{\mathcal{B}\left(r_{h}\right)}\left(16 r_{h}^{2}+a^{2} e^{\frac{7}{2} \phi\left(r_{h}\right)}\right)}{16 \pi r_{h}} .
$$

The solutions asymptote $\mathrm{AdS}_{5}$ for any value of $a$ and $T$ when the limit $r \rightarrow \infty$ is taken. In this region the metric functions and dilaton are given by

$$
\begin{aligned}
\mathcal{F}(r)= & 1+\frac{11 a^{2}}{24 r^{2}}+\frac{1}{r^{4}}\left(f_{4}-\frac{7}{12} a^{4} \log r\right)+O\left(\frac{1}{r^{6}}\right), \\
\mathcal{B}(r)= & 1-\frac{11 a^{2}}{24 r^{2}}+\frac{1}{r^{4}}\left(b_{4}+\frac{7}{12} a^{4} \log r\right)+O\left(\frac{1}{r^{6}}\right), \\
\phi(r)= & -\frac{a^{2}}{4 r^{2}}+\frac{1}{r^{4}}\left(\frac{121 a^{4}}{4032}+\frac{2 b_{4}}{7}+\frac{a^{4}}{6} \log r\right) \\
& +O\left(\frac{1}{r^{6}}\right) .
\end{aligned}
$$

The two coefficients $b_{4}$ and $f_{4}$ in Eq. (10) are not determined by the equations of motion but can be read once a numerical solution is known. It is because of this that both are functions of the anisotropy $a$ and temperature $T$ of the solution, as well as of the renormalization scale $\mu$, which will be discussed below.

The metric functions and the dilaton are known analytically in the limits of high and low temperature [33], while for intermediate regimes one has to resort to numerics to solve the equations of motion coming from Eq. (7). The explicit integration procedure is explained in detail in [33], where the solutions were computed outside the horizon. However, in order to apply the CA prescription we need to know the solutions inside the horizon, as the WDW patch extends into this region. We describe the integration procedure needed for this in Appendix A 1.

From the above discussion we can see that every member of the family of solutions is characterized by the value of its anisotropy $a$ and temperature $T$, which seem to be the only two parameters with dimensions of length, inviting us to label each solution by the dimensionless ratio $a / T$. However, it turns out that not all dimensionless physical observables are functions of this ratio alone, indicating the presence of a conformal anomaly [35] in the dual to any of the backgrounds above with $a \neq 0$. This anomaly can be explicitly exhibited by taking the trace of the stress-energy tensor in the corresponding gauge theory, obtained from the variation with respect to the boundary metric of the on-shell evaluations of the action (7). As it is usually the case in holography, the result of said evaluation diverges when the integration is taken all the way up to the boundary. To deal with this issue, the subtraction of the divergent behavior has to be done by adding covariant boundary terms to the action, in a process known as holographic renormalization $[38,39]$.

The counterterm action for the MT model is given by [33]

$$
\begin{aligned}
S_{c t}= & \frac{1}{8 \pi G_{5}}\left(\int d^{4} x \sqrt{-\gamma}\left(3-\frac{1}{8} e^{2 \phi} \partial_{i} \chi \partial^{i} \chi\right)\right. \\
& +\log r \int d^{4} x \sqrt{-\gamma} \frac{e^{4 \phi}}{12}\left(\partial_{i} \chi \partial^{i} \chi\right)^{2} \\
& \left.+\frac{1}{4}\left(C_{s c h}-1\right) \int d^{4} x \sqrt{-\gamma} \frac{e^{4 \phi}}{12}\left(\partial_{i} \chi \partial^{i} \chi\right)^{2}\right),
\end{aligned}
$$


where all the integrals are performed over a constant $r$ surface and the limit $r \rightarrow \infty$ is meant to be taken. Also, we are denoting the boundary coordinates as $x^{i}$, and all the contractions are taken using the metric $\gamma_{i j}$ induced at the boundary. The first two terms are the minimum required to eliminate the divergences of the action (7), while the third gives a contribution that remains finite in the $r \rightarrow \infty$ limit. The freedom to add such a finite term is associated with the existence of the anisotropy related conformal anomaly discussed above, which has as a consequence the introduction of an independent arbitrary energy scale $\mu$. This is the reason why some dimensionless physical quantities, such as the energy density of the system $E / T^{4}$, depend not only on the dimensionless ratio $a / T$ but also on any two independent ratios that can be built from $a, T$, and $\mu$.

In Eq. (11) we have fixed $\mu=L=1$ in the argument of the logarithm of $r$. Any change in this renormalization scale can be absorbed into the finite term by choosing a different value for the $C_{s c h}$ coefficient. We can therefore use this coefficient to reverse the change that would be introduced in physical quantities to keep a particular renormalization scheme fixed as the energy scale $\mu$ is modified, attaining as a final consequence the scheme independence of relevant physical results. It is in this sense that $C_{s c h}$ is a schemedependent quantity (see [44] for a more detailed discussion).

With the counterterms in hand, the stress-energy tensor of the system can be computed from the renormalized action

$$
S_{\text {ren }}=S+S_{c t}
$$

by taking its variation with respect to the boundary metric and evaluating the result at the boundary using the expansions (10). This procedure gives [32,33]

$$
\left\langle T_{i j}\right\rangle=\operatorname{diag}\left(E, P^{\perp}, P^{\perp}, P^{\|}\right)
$$

where $E$ is the energy of the state, while $P^{\perp}$ and $P^{\|}$are, respectively, the pressures along directions perpendicular and parallel to the anisotropic direction. These quantities are given by

$$
\begin{aligned}
E & =\mathcal{N}\left(-3 f_{4}-\frac{23}{7} b_{4}+\frac{2777}{4032} a^{4}+\frac{C_{s c h}}{24} a^{4}\right), \\
P^{\perp} & =\mathcal{N}\left(-f_{4}-\frac{5}{7} b_{4}+\frac{611}{4032} a^{4}-\frac{C_{s c h}}{24} a^{4}\right), \\
P^{\|} & =\mathcal{N}\left(-f_{4}-\frac{13}{7} b_{4}+\frac{2227}{4032} a^{4}+\frac{C_{s c h}}{8} a^{4}\right),
\end{aligned}
$$

where the explicit dependence on the three parameters $a, T$, and $\mu$ has to be supplemented by the one implicit in $f_{4}$ and $b_{4}$ that was previously discussed, and the normalization constant $\mathcal{N}$ is given by

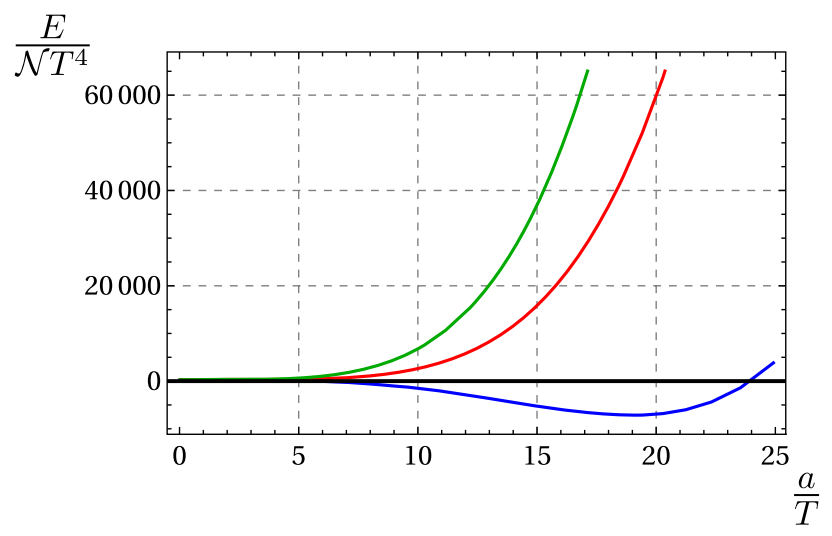

FIG. 1. Energy density for the MT model as a function of $a / T$. Blue, red, and green curves (bottom to top) correspond to $C_{\text {sch }}=\{-10,0,10\}$, respectively.

$$
\mathcal{N}=\frac{V_{x}}{16 \pi G_{5}}
$$

with $V_{x}$ the spatial volume of the boundary region under consideration. From this expression we can see that the trace of the stress-energy tensor is

$$
\left\langle T^{i}{ }_{i}\right\rangle=\frac{\mathcal{N}}{6} a^{4},
$$

which does not vanish for any $a \neq 0$, showing the existence of the conformal anomaly. In Fig. 1 we show the quantity $E / \mathcal{N} T^{4}$ in the MT model as a function of $a / T$ for three different values of $C_{s c h}$, as we do not have any renormalization conditions to fix the scheme at this stage.

\section{B. Magnetic black branes}

The DK model [34] is a family of solutions to the fivedimensional gauged supergravity whose bosonic part of the action is given by

$$
\begin{aligned}
S= & \frac{1}{16 \pi G_{5}}\left[\int d^{5} x \sqrt{-g}\left(R-F^{2}+\frac{12}{L^{2}}\right)\right. \\
& \left.+\frac{8}{3 \sqrt{3}} \int A \wedge F \wedge F+\int d x^{4} \sqrt{-\gamma} 2 K\right],
\end{aligned}
$$

where $F$ and $g_{\mu \nu}$ are, respectively, the Maxwell and metric fields, $G_{5}$ is the five-dimensional Newton constant, and $L$ is the $\mathrm{AdS}_{5}$ radius, which we will set again to unity without loss of generality. The last integral is the YGH surface term, in which $\gamma_{i j}$ is the induced metric at the boundary and $K$ its extrinsic curvature. This theory is a consistent truncation of ten-dimensional type IIB SUGRA [45], and the backgrounds that we are about to study were uplifted in [46] to turn them into configurations that solve the proper equations of the latter theory. 
Every member of the DK family of solutions is part of the Ansatz.

$$
\begin{aligned}
d s^{2} & =\frac{d r^{2}}{U(r)}-U(r) d t^{2}+V(r)\left(d x^{2}+d y^{2}\right)+W(r) d z^{2} \\
F & =b d x \wedge d y
\end{aligned}
$$

where $r$ is the $\mathrm{AdS}_{5}$ radial coordinate, in terms of which the boundary is located at $r \rightarrow \infty$. All these backgrounds feature a horizon located at $r_{h}$ where the metric function $U(r)$ vanishes. The magnetic field intensity $b$ matches the one in the dual gauge theory given that the metric asymptotes precisely $\mathrm{AdS}_{5}$ at the boundary. From the ten-dimensional perspective this Maxwell field is interpreted as an infinitesimal rotation in the compact part of the geometry (see [45,47] for additional details). Meanwhile, the temperature of each solution is given by

$$
T=\frac{3 r_{h}}{2 \pi}
$$

Thus, every member of the family is characterized by the values of its magnetic field intensity $b$ and temperature $T$, which at first sight seem to be the only two parameters with dimensions of length, suggesting the labeling of each solution by the dimensionless ratio $b / T^{2}$.

The only known analytical members of this family are the Schwarzschild-AdS black brane for $b / T^{2}=0$ and $\mathrm{BTZ} \times \mathbb{R}^{2}$ for precisely $b / T^{2}=\infty$. For any intermediate values it is necessary to resort to numerical methods to solve the equations coming from Eq. (17). The explicit integration procedure that we follow is explained in detail in [48] for the exterior solutions, and in [49] for the interior solutions. We review both in Appendix A 2. The backgrounds are constructed so that for any value of $b / T^{2}$ the geometry asymptotes $\mathrm{AdS}_{5}$ when the limit $r \rightarrow \infty$ is taken. In this region the functions in the line element (18) are given by

$$
\begin{aligned}
& U(r)=r^{2}+u_{1} r+\frac{u_{1}^{2}}{4}+\frac{1}{r^{2}}\left(u_{4}-\frac{2}{3} b^{2} \log r\right)+\mathcal{O}\left(\frac{1}{r^{4}}\right), \\
& V(r)=r^{2}+u_{1} r+\frac{u_{1}^{2}}{4}+\frac{1}{r^{2}}\left(-\frac{1}{2} w_{4}+\frac{1}{3} b^{2} \log r\right)+\mathcal{O}\left(\frac{1}{r^{4}}\right), \\
& W(r)=r^{2}+u_{1} r+\frac{u_{1}^{2}}{4}+\frac{1}{r^{2}}\left(w_{4}-\frac{2}{3} b^{2} \log r\right)+\mathcal{O}\left(\frac{1}{r^{4}}\right) .
\end{aligned}
$$

The three coefficients $u_{1}, w_{4}$, and $u_{4}$ are not determined by the equations of motion but can be read from each numerical solution. Consequently all three coefficients are functions of the magnetic field $b$ and the temperature $T$ of the background, through dimensionless combinations involving the renormalization scale $\mu$ that will be introduced below.

While it would seem like any member of the family of solutions can be characterized by the dimensionless ratio $b / T^{2}$, not every physical observable is solely a function of this ratio. Just like the MT model, the dual of any solution with nonvanishing magnetic field $b \neq 0$ features a conformal anomaly. In order to obtain the stress-energy tensor required to support the latter claims, it is necessary to compute the on-shell action (17) and take its variation with respect to the boundary metric. The result of this once again diverges when the integration is taken all the way up to the boundary.

The counterterm action for the DK model is given by [50,51]

$S_{c t}=-\frac{1}{16 \pi G_{5}} \int \sqrt{-\gamma}\left(6-F^{i j} F_{i j} \log r+C_{s c h} F^{i j} F_{i j}\right)$,

where the integral is performed over a constant $r$ surface and the limit $r \rightarrow \infty$ is meant to be taken. The first two terms are the minimum required to remove the divergences in the action (7), while the third gives a finite contribution in the limit $r \rightarrow \infty$. As discussed previously for the MT model, the freedom to add a finite term to the action is related to the existence of a conformal anomaly in the theory, which introduces an arbitrary energy scale $\mu$. We fixed this scale to $\mu=L=1$ in Eq. (21).

The stress-energy tensor of the system can be computed from the renormalized action

$$
S_{\text {ren }}=S+S_{c t}
$$

by taking its variation with respect to the boundary metric and evaluating the result at the boundary using the expansions (20). The result of doing this is $[50,51]$

$$
\left\langle T_{i j}\right\rangle=\operatorname{diag}\left(E, P^{\perp}, P^{\perp}, P^{\|}\right),
$$

where $E$ is the energy density of the state in the gauge theory, while $P^{\perp}$ and $P^{\|}$are, respectively, the pressures along directions perpendicular and parallel to the magnetic field. This quantities are explicitly given by

$$
\begin{aligned}
E & =\mathcal{N}\left(-3 u_{4}-2 C_{s c h} b^{2}\right), \\
P^{\perp} & =\mathcal{N}\left(-u_{4}-2 w_{4}-b^{2}\left(1+2 C_{s c h}\right)\right), \\
P^{\|} & =\mathcal{N}\left(-u_{4}+4 w_{4}+2 C_{s c h} b^{2}\right),
\end{aligned}
$$

where $u_{4}$ and $w_{4}$ depend on $b, T$, and $\mu$ as previously discussed, and the normalization constant $\mathcal{N}$ is the same as in Eq. (15). Using these expressions we can take the trace of the stress-energy tensor to find 


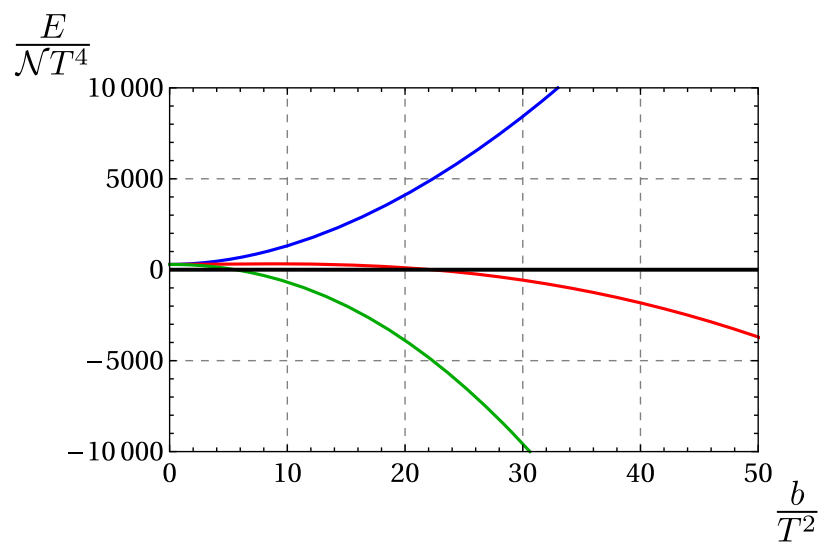

FIG. 2. Energy density for the DK model as a function of $b / T^{2}$. Blue, red, and green curves (top to bottom) correspond to $C_{s c h}=\{-5,0,5\}$, respectively.

$$
\left\langle T^{i}{ }_{i}\right\rangle=-2 \mathcal{N} b^{2}
$$

which is nonvanishing for any $b \neq 0$, showing the existence of the conformal anomaly. In Fig. 2 we show the quantity $E / \mathcal{N} T^{4}$ in the DK model as a function of $b / T^{2}$ for three different values of $C_{s c h}$.

\section{Penrose diagram}

In this section we will derive the Penrose diagram for both the MT and DK models. The metric Ansätze for both are of the form

$$
\begin{aligned}
d s^{2}= & g_{r r}(r) d r^{2}+g_{t t}(r) d t^{2}+g_{x x}(r) d x^{2} \\
& +g_{y y}(r) d y^{2}+g_{z z}(r) d z^{2}
\end{aligned}
$$

where we are including the gauge theory spatial directions, even if they are not relevant to the derivation of the Penrose diagram. That being said, it is important to know that the metric functions $g_{r r}$ and $g_{t t}$ strongly depend on the source of the anisotropy, either $a$ or $b$. These two functions are such that $g_{t t}$ has a zero at $r_{h}$, while $g_{r r}$ has a simple pole there [52].

In order to construct the Penrose diagram for the metric (26) we first change to the tortoise coordinate $r_{\star}$, which is given by the solution to the equation

$$
\frac{d r_{\star}}{d r}=\operatorname{sign}\left(g_{r r}\right) \sqrt{\left|\frac{g_{r r}}{g_{t t}}\right|},
$$

which satisfies the boundary condition $r_{\star}(\infty)=0$. Note that this coordinate automatically satisfies $r_{\star} \rightarrow \log (r-$ $\left.r_{h}\right)$ as $r \rightarrow r_{h}$ because of the simple pole of $g_{r r}$ and the zero of $g_{t t}$ at $r_{h}$. Next we transform to the Kruskal-Szekeres coordinates, which are given by

$$
\begin{array}{lll}
\mathcal{U}=+e^{-2 \pi T\left(t-r_{\star}\right)}, & \mathcal{V}=-e^{2 \pi T\left(t+r_{\star}\right)} & \text { (left exterior), } \\
\mathcal{U}=-e^{-2 \pi T\left(t-r_{\star}\right)}, & \mathcal{V}=+e^{2 \pi T\left(t+r_{\star}\right)} & \text { (right exterior), } \\
\mathcal{U}=+e^{-2 \pi T\left(t-r_{\star}\right)}, & \mathcal{V}=+e^{2 \pi T\left(t+r_{\star}\right)} & \text { (future interior), } \\
\mathcal{U}=-e^{-2 \pi T\left(t-r_{\star}\right)}, & \mathcal{V}=-e^{2 \pi T\left(t+r_{\star}\right)} & \text { (past interior), }
\end{array}
$$

where $T$ is the temperature of the black hole. Finally, we change to the compact coordinates

$X=\frac{\arctan \mathcal{V}-\arctan \mathcal{U}}{2}, \quad Y=\frac{\arctan \mathcal{V}+\arctan \mathcal{U}}{2}$,

which leave the metric as

$d s^{2}= \pm \frac{g_{t t}(X, Y) e^{-4 \pi T r_{\star}}}{4(\pi T)^{4}}\left(1+\mathcal{V}^{2}\right)\left(1+\mathcal{U}^{2}\right)\left(-d Y^{2}+d X^{2}\right)$

We present the Penrose diagram for both models, at different values of $a / T$ and $b / T^{2}$, in Figs. 4 and 6 , respectively. Note that in the case of the DK model in Fig. 6 the position of the singularity changes as $b / T^{2}$ increases, as explained in Appendix A 2. For the following analysis it is also relevant to notice that the hypersurface $t=0$ corresponds to a horizontal line in the middle of the Penrose diagram of either model.

\section{WDW PATCH}

The WDW patch is the region enclosed by past and future light sheets extending into the bulk from a constant time slice on the boundary, where the complexity is meant to be evaluated. Because the TFD state depends on time only through the combination $t_{L}+t_{R}$, without loss of generality we will adopt the convention $t_{L}=t_{R}=t_{0}$ [53]. The direct evaluation of the action on the WDW patch is divergent, as it extends to the boundary at $r=\infty$ and to the singularity at $r=r_{s}$. To avoid this, we regularize it by introducing the cutoffs $r_{\max }$ near the boundary and $r_{\text {min }}$ near the singularity. At the end of the calculation we will remove these regulators by taking the limits $r_{\max } \rightarrow \infty$ and $r_{\text {min }} \rightarrow r_{s}$.

For a given boundary time $t_{0}$, the light sheets that delimit the WDW patch are given by

$$
\begin{aligned}
& r_{\star}(r)+t=t_{0} \quad \text { (right future light sheet), } \\
& r_{\star}(r)-t=-t_{0} \quad \text { (right past light sheet), } \\
& r_{\star}(r)-t=t_{0} \quad \text { (left future light sheet), } \\
& r_{\star}(r)+t=-t_{0} \quad \text { (left past light sheet). }
\end{aligned}
$$


The names are determined by which of the singularities the light sheet reaches for $t_{0}=0$. Note that the difference in signs between the left and right regions comes from the fact that in the left region the flow of time is reversed, but we are taking $t_{0}=t_{L}=t_{R}$ by definition.

At early times, close to $t_{0}=0$, the WDW patch intersects both the past and future singularities, which, as we will see in the following, makes its volume constant for some period of time $0 \leq t_{0} \leq t_{c}$. However, for later times $t_{0}>t_{c}$, the WDW patch no longer intersects the past singularity and its volume reduces as time passes. This $t_{c}$ is known as the critical time and is given by

$$
t_{c}=-r_{\star}\left(r_{s}\right) \text {, }
$$

where we used the equations for the past light sheets in Eq. (31) to find the $t_{0}$ for which they intersect exactly at the singularity. Thus, for $0 \leq t_{0} \leq t_{c}$ and any $(x, y, z)$ we can naturally divide the WDW patch into four regions:

$$
\begin{aligned}
\mathrm{I} & =\left\{(t, r) \mid r \in\left[r_{\text {min }}, r_{h}\right], t \in\left[-t_{0}+r_{\star}(r), t_{0}-r_{\star}(r)\right]\right\}, \\
\mathrm{II} & =\left\{(t, r) \mid r \in\left[r_{h}, r_{\text {max }}\right], t \in\left[t_{0}+r_{\star}(r), t_{0}-r_{\star}(r)\right]\right\}, \\
\mathrm{III} & \left.=\left\{(t, r) \mid r \in\left[r_{\text {min }}, r_{h}\right], t \in\left[t_{0}+r_{\star}(r)\right),-t_{0}-r_{\star}(r)\right]\right\}, \\
\mathrm{IV} & =\left\{(t, r) \mid r \in\left[r_{h}, r_{\max }\right], t \in\left[-t_{0}+r_{\star}(r),-t_{0}-r_{\star}(r)\right]\right\} .
\end{aligned}
$$

We illustrate these regions in the Penrose diagram in Fig. 4(a) for the MT model and in Fig. 6(b) for the DK model.

For later times the WDW patch does not reach the past singularity and instead ends on a minimal radius $r_{m}$ given by the intersection of the left and right past light sheets. Thus, it is defined implicity by the solution of the equation

$$
r_{\star}\left(r_{m}\right)=-t_{0}
$$

as a function of the fixed boundary time $t_{0}$. It will be useful to have an expression for the derivative of $r_{m}$ with respect to $t_{0}$. We can manipulate the derivative of the previous expression to get

$$
\frac{d r_{m}}{d t_{0}}=-\left.\operatorname{sign}\left(g_{r r}\right) \sqrt{\left|\frac{g_{t t}}{g_{r r}}\right|}\right|_{r=r_{m}},
$$

where we have used Eq. (27). The critical time for Schwarzschild-AdS 5 can be computed analytically, and the result is

$$
t_{c}=\frac{1}{4 T} .
$$

We show the WDW patch at $t_{0}>t_{c}$ in the Penrose diagram for the MT model in Fig. 4(b) and for the DK model in Fig. 6(b). The definitions of regions I, II, and IV are still given by Eq. (33), while the one for region III changes to

$$
\mathrm{III}=\left\{(t, r) \mid r \in\left[r_{m}\left(t_{0}\right), r_{h}\right], t \in\left[t_{0}+r_{\star}(r),-t_{0}-r_{\star}(r)\right]\right\} .
$$

\section{A. MT model}

The behavior of the critical time as a function of the anisotropic parameter $a / T$ is shown in Fig. 3. From this it can be seen that $t_{c}(a) / t_{c}(0)$ decreases as $a / T$ increases or, in other words, that the anisotropy has the effect of making the WDW patch withdraw from the past singularity at earlier times than in the $a=0$ case. This result coincides with what was found in [31] for $a / T \ll 1$.

In Fig. 4 we present the Penrose diagram and the WDW patch for the MT model at $a / T=242$. Figure 4(a) corresponds to $t_{0} / t_{c}=0$, which is an early-time configuration because it satisfies $t_{0}<t_{c}$, while Fig. 4(b) corresponds to $t_{0} / t_{c}=1.86$, which is a late-time configuration with $t_{0}>t_{c}$. In both cases the red and blue lines represent the right and left light sheets, respectively, the points denote the joints, and the dashed curves correspond to the boundary regulator at $r=r_{\max }$. We also show explicitly the four regions defined in Eqs. (33) and (37): I (dark blue) is the future interior, II (red) is the right exterior, III (green) is the past interior, and IV (light blue) is the left exterior. It can be seen that in Fig. 4(a) the WDW patch reaches the past singularity, and thus there is a total of eight joints to consider in the evaluation of the holographic complexity. On the other hand, Fig. 4(b) shows that for $t_{0}>t_{c}$ the WDW patch no longer reaches the past singularity, and thus there is now a total of seven joints.

\section{B. DK model}

We show in Fig. 5 how the critical time depends on the magnetic field intensity $b / T^{2}$ for the DK model. From this

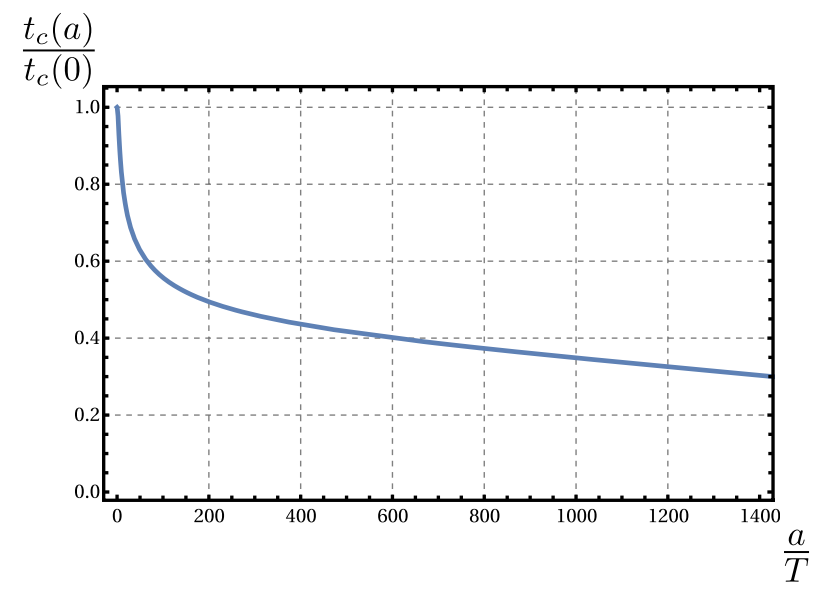

FIG. 3. Critical time $t_{c}(a) / t_{c}(0)$ as a function of $a / T$ for the MT model. 


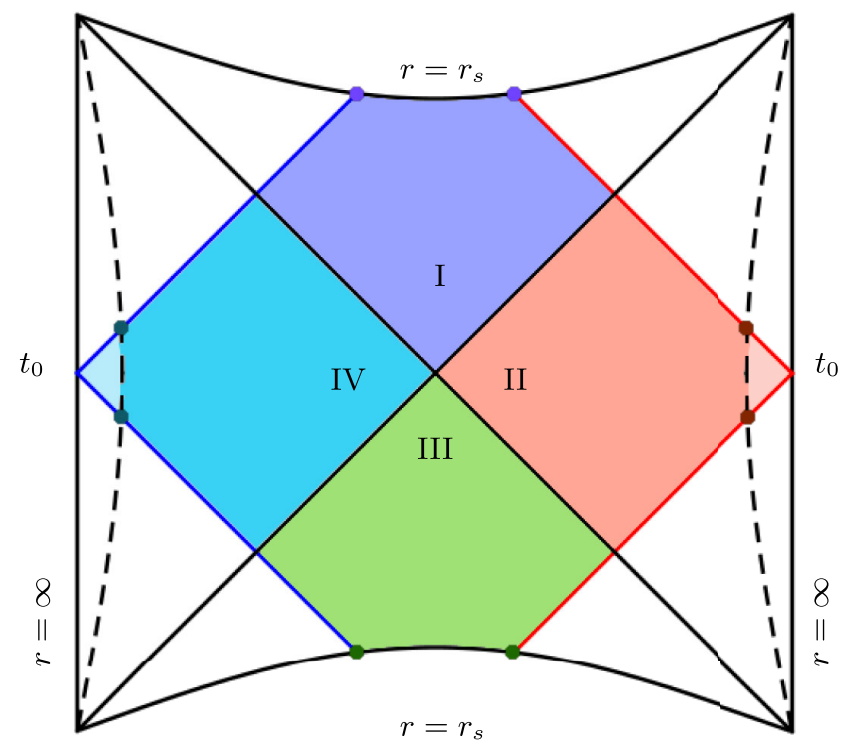

(a)

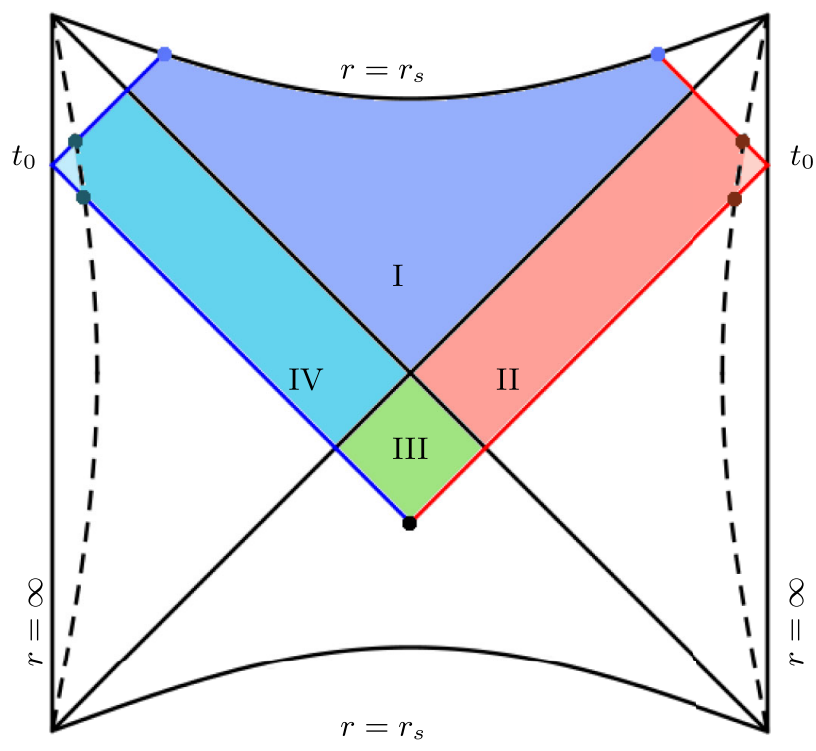

(b)

FIG. 4. Penrose diagram and WDW patch for the MT model. (a) Configuration with $t_{0} / t_{c}=0$ showing that the WDW patch reaches both the future and past singularities. (b) Late-time configuration with $t_{0} / t_{c}=1.86$. It can be seen that the WDW patch no longer reaches the past singularity. In both cases the red and blue lines represent the right and left light sheets, respectively, the points denote the joints, and the dashed curve corresponds to the boundary regulator at $r=r_{\max }$. The four regions of the WDW patch are displayed: I (dark blue) is the future interior, II (red) is the right exterior, III (green) is the past interior, and IV (light blue) is the left exterior. In both figures the anisotropy is $a / T=242$.

it can be seen that $t_{c}(b) / t_{c}(0)$ decreases as $b / T^{2}$ increases, that is, like the anisotropy in the MT model, the magnetic field has the effect of making the WDW patch withdraw from the past singularity at earlier times relative to the $b=0$ case.

In Fig. 6 we show the Penrose diagram and the WDW patch for the DK model at $b / T^{2}=152.20$. Fig. 6(a) corresponds to an early time $t_{0}<t_{c}$ given by $t_{0} / t_{c}=0$, while Fig. 6(b) corresponds to $t / t_{c}=3.22$, which is a latetime configuration with $t_{0}>t_{c}$. Note that, as mentioned in Sec. II B, the position of the singularity in the $r$ coordinate

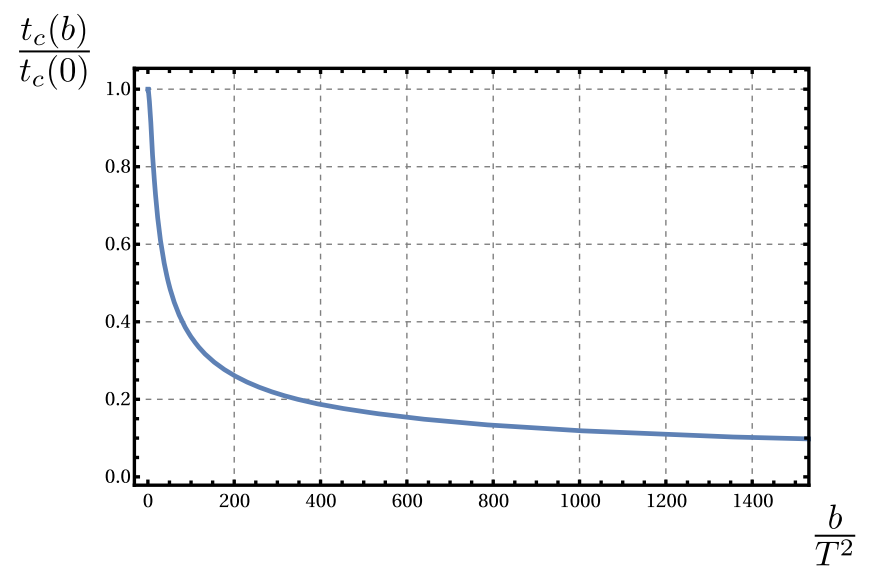

FIG. 5. Critical time $t_{c}(b) / t_{c}(0)$ as a function of $b / T^{2}$ for the DK model. changes with the magnetic field. For $b / T^{2}=152.20$, the singularity is located at $r_{s} / r_{h}=-0.06$. As before, the red and blue lines represent the right and left light sheets, respectively, the points denote the joints, and the dashed curves corresponds to the boundary regulator at $r=r_{\max }$. The Penrose diagram conveniently displays the four regions defined in Eqs. (33) and (37): I (dark blue) is the future interior, II (red) is the right exterior, III (green) is the past interior, and IV (light blue) is the left exterior. Just as in the MT model, it can be seen in Fig. 6(a) that the WDW patch reaches the past singularity, and thus there is a total of eight joints to consider in the evaluation of the holographic complexity. On the other hand, Fig. 6(b) shows that for $t_{0}>t_{c}$ the WDW patch no longer reaches the past singularity, and thus there is now a total of seven joints.

\section{HOLOGRAPHIC COMPLEXITY}

In this section we compute the holographic complexity using the CA prescription. We closely follow the analysis presented in [31] but offer additional details regarding the particularities of our numerical solutions, such as the conformal anomaly. As previously explained, the evaluation of the action over the WDW patch requires the addition of extra boundary terms to make sure that the variational principle is well posed. Thus, the full action has three types of contributions: one related to the bulk, another related to boundary surfaces, and the last one for the joints between these surfaces, i.e., 


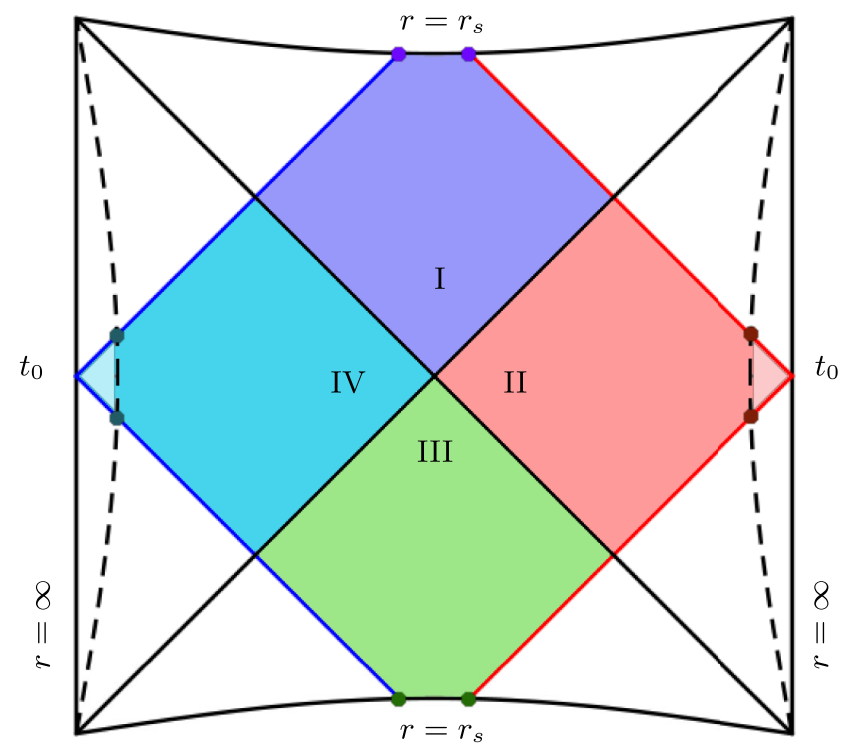

(a)

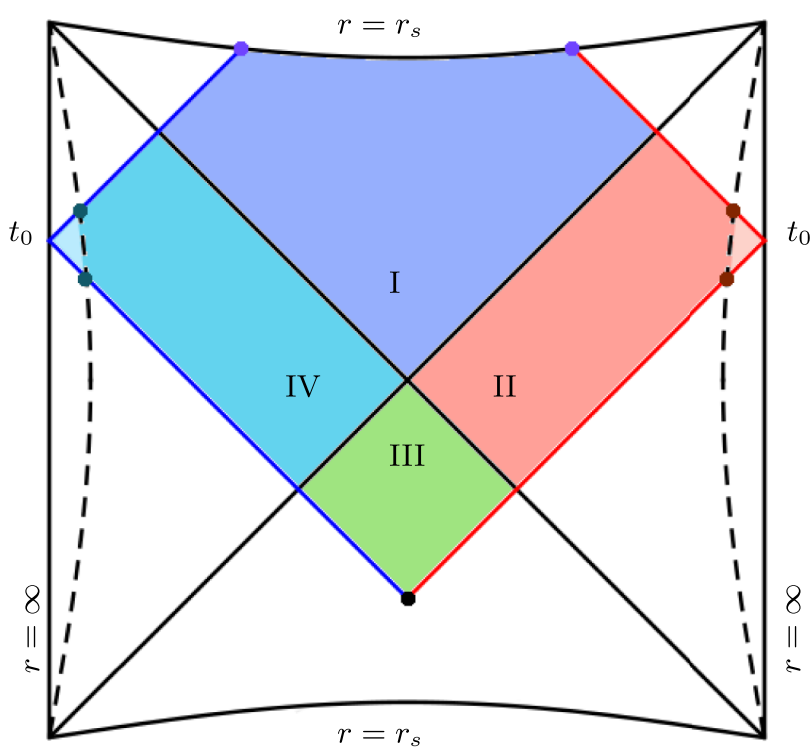

(b)

FIG. 6. Penrose diagram and WDW patch for the DK model. (a) Early-time configuration with $t_{0} / t_{c}=0$. The WDW patch reaches both the future and past singularities. (b) Late-time configuration with $t_{0} / t_{c}=3.22$. The WDW patch no longer reaches the past singularity. In both cases the red and blue lines represent the right and left light sheets, respectively, the points denote the joints, and the dashed curve corresponds to the boundary regulator at $r=r_{\max }$. The four regions of the WDW patch are displayed: I (dark blue) is the future interior, II (red) is the right exterior, III (green) is the past interior, and IV (light blue) is the left exterior. In both figures the magnetic field intensity is $b / T^{2}=152.20$, which means that the singularity is located at $r_{s} / r_{h}=-0.06$.

$$
S_{\mathrm{WDW}}=S_{\text {bulk }}+S_{\text {surface }}+S_{\text {joint }}
$$

where the schematic form of each term is

$$
\begin{aligned}
S_{\text {bulk }} & =\frac{1}{16 \pi G_{5}} \int_{\mathcal{M}} d^{5} x \sqrt{-g} \mathcal{L}, \\
S_{\text {surface }} & =\frac{1}{16 \pi G_{5}} \int_{\mathcal{B}} d^{4} x \sqrt{|\gamma|} 2 K+\frac{1}{16 \pi G_{5}} \int_{\mathcal{B}^{\prime}} d \lambda d^{3} \theta \sqrt{h} 2 \kappa \\
S_{\text {joint }} & =\frac{1}{16 \pi G_{5}} \int_{\Sigma^{\prime}} d^{3} x \sqrt{\sigma} 2 a .
\end{aligned}
$$

The first expression in Eq. (39) is the bulk action of the theory, where $\mathcal{M}$ refers to the interior of the WDW patch. The first integral on the right-hand side on the second line of Eq. (39) is the YGH surface term for the spacelike and timelike segments $\mathcal{B}$ of the boundary of the WDW patch, which is written using the induced metric $\gamma$ and the extrinsic curvature $K$. The second integral on the same expression is the surface term for the null segment $\mathcal{B}^{\prime}$ of the boundary of the WDW patch, defined by the induced metric $h$ and the function $\kappa$, which measures the failure of the null generators to be affinely parametrized. Finally, $S_{\text {joint }}$ denotes the joint terms, where $\sigma$ is the determinant of the induced metric on this codimension- 2 surface. The integrand of this expression takes different forms depending on which kind of intersection is considered. For the three possible cases we explicitly have

$$
a= \begin{cases}\epsilon \log |\boldsymbol{t} \cdot \boldsymbol{k}| & \text { for space-null joint with } \\ & \epsilon=-\operatorname{sign}(\boldsymbol{t} \cdot \boldsymbol{k}) \operatorname{sign}(\hat{s} \cdot \boldsymbol{k}), \\ \epsilon \log |\boldsymbol{s} \cdot \boldsymbol{k}| & \text { for time-null joint with } \\ & \epsilon=-\operatorname{sign}(\boldsymbol{s} \cdot \boldsymbol{k}) \operatorname{sign}(\hat{t} \cdot \boldsymbol{k}), \\ \epsilon \log \left|\frac{\boldsymbol{k}_{1} \cdot \boldsymbol{k}_{2}}{2}\right| & \text { for null-null joint with } \\ & \epsilon=-\operatorname{sign}\left(\boldsymbol{k}_{\mathbf{1}} \cdot \boldsymbol{k}_{\mathbf{2}}\right) \operatorname{sign}\left(\hat{k}_{1} \cdot \boldsymbol{k}_{\mathbf{2}}\right),\end{cases}
$$

where $\boldsymbol{s}, \boldsymbol{t}$, and $\boldsymbol{k}$ are the normalized outward unit normal one-forms to the spacelike, timelike, and null surfaces under consideration, respectively, while $\boldsymbol{k}_{\mathbf{1}}$ and $\boldsymbol{k}_{\mathbf{2}}$ are two normal outward one-forms to different but intersecting null surfaces. The sign $\epsilon$ in each case is determined by the auxiliary vectors $\hat{s}, \hat{t}$, and $\hat{k_{1}}$, which are normal to the spacelike, timelike, and null surfaces, respectively. We will explicitly write this vectors and one-forms below, following the procedure and conventions outlined in [54].

As first explained in [18], there are certain ambiguities associated with the null surface contributions. In particular, the action depends on the parametrization chosen for the null generators as, for example, an affine parametrization 
sets $\kappa=0$ and eliminates the null surface contributions from the action altogether. In order to remove this ambiguity, it is necessary to add the counterterm

$$
S_{\text {null }}=\frac{1}{16 \pi G_{5}} \int d \lambda d^{3} \theta \sqrt{h} 2 \Theta \log \left(l_{\text {null }} \Theta\right),
$$

where $\Theta$ is the expansion of the null generators defined as

$$
\Theta=\partial_{\lambda} \log (\sqrt{h}) .
$$

While Eq. (41) is by itself dependent on the parametrization of the null generators, the full action turns out to be invariant when adding it. Thus, we will follow the usual conventions and chose an affine $\lambda$. Also note that $S_{\text {null }}$ introduces an arbitrary length scale $l_{\text {null }}$, which is related to the freedom of choosing a reference state in the dual theory [55]. We will leave it arbitrary and show that it does not modify the late-time behavior of the rate of change of the complexity for the type of geometries that we consider.

The inclusion of this term does not affect certain aspects of the complexity, such as the complexity of formation [56] or the late-time behavior of the complexity in the case of the eternal black hole [19]. However, it can modify other aspects [57] like the structure of the UV divergences $[55,58]$. Here we will consider $S_{\text {null }}$ to determine whether it is affected by the presence of the conformal anomaly.

\section{A. Before the critical time: $0 \leq t_{0} \leq t_{c}$}

We first compute the bulk contribution. In order to do this, we need to evaluate the action on each of the four regions that constitute the WDW patch,

$$
S_{\text {bulk }}=S_{I}+S_{I I}+S_{I I I}+S_{I V} \text {. }
$$

Given that the metric and the Lagrangian depend only on $r$, when evaluating $S_{\text {bulk }}$ we can integrate along the $(x, y, z)$ directions and obtain an overall factor of $V_{x}$. The integration over time is also easily performed using the definition of each region given in Eq. (33). Thus, we have

$$
\begin{gathered}
S_{I}=2 \mathcal{N} \int_{r_{\text {min }}}^{r_{h}} d r \sqrt{-g} \mathcal{L}(r)\left(t_{0}-r_{\star}(r)\right), \\
S_{I I}=S_{I V}=-2 \mathcal{N} \int_{r_{h}}^{r_{\max }} d r \sqrt{-g} \mathcal{L}(r) r_{\star}(r), \\
S_{I I I}=2 \mathcal{N} \int_{r_{\text {min }}}^{r_{h}} d r \sqrt{-g} \mathcal{L}(r)\left(-t_{0}-r_{\star}(r)\right),
\end{gathered}
$$

and by substituting them into Eq. (43) we obtain

$$
S_{\text {bulk }}\left(0 \leq t_{0} \leq t_{c}\right)=-4 \mathcal{N} \int_{r_{\min }}^{r_{\max }} d r \sqrt{-g} \mathcal{L}(r) r_{\star}(r) .
$$

At this stage we note two important things. The first is that the $t_{0}$ dependence has been completely eliminated from Eq. (45). This is because the volume of the WDW patch remains constant for any $0 \leq t_{0} \leq t_{c}$, and any member of the family of solutions of either the MT or DK model is static. The second is that this integral is divergent as the limit $r_{\max } \rightarrow \infty$ is taken.

Next we turn to the surface integrals. As previously explained, by choosing an affine parameter we eliminate the contribution coming from the second term on the second line of Eq. (39). Hence, we are left with the integrals at the two spacelike and two timelike surfaces at $r=r_{\max }$ and $r=r_{\min }$, respectively. Schematically we have

$$
S_{\text {surface }}=S_{\text {future }}+S_{\text {right }}+S_{\text {past }}+S_{\text {left }},
$$

where $S_{\text {future }}$ denotes the integral evaluated at the regulator near the future singularity, $S_{\text {past }}$ is the analogous term for the past singularity, and $S_{\text {right }}$ and $S_{\text {left }}$ correspond to the integrals at the regulators near the right and left boundaries, respectively. In all cases the integral to consider is the YGH term, given by the induced metric in the surface $\gamma_{i j}$ and the extrinsic curvature

$$
K_{i j}=\frac{\partial x^{\mu}}{\partial y^{i}} \frac{\partial x^{\nu}}{\partial y^{j}} \nabla_{\mu} n_{\nu}, \quad K=\gamma^{i j} K_{i j},
$$

where $y^{i}$ are the coordinates on the surface defined by $x^{\mu}=$ $x^{\mu}\left(y^{i}\right)$ and $n_{\mu}$ are the components of the corresponding unit outward normal one-form. The latter are explicitly given by

$$
\begin{aligned}
& \boldsymbol{s}=\sqrt{\left|g_{r r}\right|} d r \quad \text { for } r=r_{\max }, \\
& \boldsymbol{t}=-\sqrt{\left|g_{r r}\right|} d r \quad \text { for } r=r_{\min } .
\end{aligned}
$$

Applying the previous expressions for both the MT and DK models, it can be shown that the integrand of the YGH term is a function of $r$ only,

$$
2 \sqrt{|\gamma|} K= \pm \mathcal{G}(r)
$$

where the plus sign is used for the $r=r_{\max }$ surfaces and the minus sign is used for the near singularity regulators at $r=r_{\min }$. Using this fact we can easily evaluate the surface integrals, which gives

$$
\begin{aligned}
S_{\text {future }} & =-2 \mathcal{N} \mathcal{G}\left(r_{\text {min }}\right)\left(t_{0}-r_{\star}\left(r_{\text {min }}\right)\right), \\
S_{\text {right }} & =S_{\text {left }}=-2 \mathcal{N} \mathcal{G}\left(r_{\text {max }}\right) r_{\star}\left(r_{\text {max }}\right), \\
S_{\text {past }} & =-2 \mathcal{N} \mathcal{G}\left(r_{\text {min }}\right)\left(-t_{0}-r_{\star}\left(r_{\text {min }}\right)\right),
\end{aligned}
$$

and thus after substitution into Eq. (46) the result is

$$
S_{\text {surface }}\left(0 \leq t_{0} \leq t_{c}\right)=-4 \mathcal{N} \mathcal{G}(r) r_{\star}(r) \mid r_{\min } r_{\max } .
$$


Note that once again, this contribution is independent of $t_{0}$. This is because the $r_{\max }$ surfaces suffer only a time translation, while the area in which the future $r_{\min }$ surface gains is exactly the area in which the past $r_{\min }$ one loses. In other words, the $t_{0}$ dependence of $S_{\text {future }}$ exactly cancels the one coming from $S_{\text {past }}$. Also note that $S_{\text {surface }}$ diverges as the limit $r_{\max } \rightarrow \infty$ is taken.

Next we have the contribution coming from the joint terms. As can be seen in Figs. 4(a) and 6(a), there are eight joints to consider for $0 \leq t_{0} \leq t_{c}$, and all of them are of the space null or the time null type. In order to evaluate $S_{\text {joint }}$ we need to define the auxiliary vectors appearing in Eq. (40). As explained in [54], these vectors need to live in the tangent space of the corresponding surface, be orthogonal to the joint, and point outward to the WDW patch. Note that these vectors do not need to be normalized, as can be seen from the way they enter into Eq. (40). For the space-null joints we have

$$
\hat{s}_{ \pm}= \pm \partial_{t}
$$

where the plus sign is used for the joints at the future singularity regulator, and the minus sign for the ones at the past singularity regulator. On the other hand, for the timenull joints we use

$$
\hat{t}_{ \pm}= \pm \partial_{t}
$$

where the plus sign is used for the joints at the future light sheets, while the minus sign is used for the joints at the past light sheets. We also need the outward normal one-forms to the null hypersurfaces, which are specified by an expression of the form $\Phi\left(x^{\mu}\right)=0$ [explicitly given in Eq. (31)]. Thus, the normal one-forms are given by $\alpha d \Phi$, where $\alpha>0$ is an arbitrary but positive normalization constant. While this is another ambiguity that comes from the fact that the WDW patch contains null segments, as we will see below this arbitrary constant affects the value of the complexity of the state, but the late-time behavior of the rate of change of the complexity is unaffected by it (see $[18,19,54]$ for a detailed discussion). For each light sheet we have

$$
\boldsymbol{k}_{\mathbf{1}}=\alpha\left(\operatorname{sign}\left(g_{r r}\right) \sqrt{\left|\frac{g_{r r}}{g_{t t}}\right|} d r+d t\right)
$$

for the right future and left past light sheets, and

$$
\boldsymbol{k}_{\mathbf{2}}=\alpha\left(\operatorname{sign}\left(g_{r r}\right) \sqrt{\left|\frac{g_{r r}}{g_{t t}}\right|} d r-d t\right)
$$

for the right past and left future light sheets.

After substituting Eqs. (48), (52), (53), and (55) into Eq. (40), for the joints in regions I and III we have

$$
S_{\text {joint }}=\left.2 \mathcal{N} \sqrt{\sigma} \log \left(\frac{\alpha}{\sqrt{\left|g_{t t}\right|}}\right)\right|_{r=r_{\text {min }}},
$$

while for the joints in regions II and IV we have

$$
S_{\text {joint }}=-\left.2 \mathcal{N} \sqrt{\sigma} \log \left(\frac{\alpha}{\sqrt{\left|g_{t t}\right|}}\right)\right|_{r=r_{\max }} .
$$

Hence, the full joint contribution is

$$
S_{\text {joint }}\left(0 \leq t_{0} \leq t_{c}\right)=-\left.8 \mathcal{N} \sqrt{\sigma} \log \left(\frac{\alpha}{\sqrt{\left|g_{t t}\right|}}\right)\right|_{r_{\min }} ^{r_{\max }} .
$$

Note also that $S_{\text {joint }}$ is independent of $t_{0}$.

Finally, we have the contribution coming from the null counterterm (41). The generators for each hypersurface are given by Eq. (31); hence, we parametrize the null surfaces with $\theta^{i}=(x, y, z)$ and the induced metric is given by

$$
h_{A B}=g_{\alpha \beta} \frac{d x^{\alpha}}{d \theta^{A}} \frac{d x^{\beta}}{d \theta^{B}} .
$$

From Eq. (59) we see that for metrics with the structure of Eq. (26) that we are considering, $h_{A B}$ is equal to the induced metric on the joints $\sigma_{A B}$. To make the above consistent with our previous calculations, we need to choose an affine parameter for the null generators. A direct substitution of the normal vectors as written in Eq. (55) in the geodesic equation yields

$$
k^{\alpha} \nabla_{\alpha} k_{\mu}=0
$$

for any constant $\alpha>0$. Hence, when we use the fact that

$$
k^{\mu}=\frac{d x^{\mu}}{d \lambda}
$$

the affine parameter $\lambda$ is given by the solution to the equations

$$
\frac{d r}{d \lambda}=\frac{\alpha}{\sqrt{\left|g_{r r} g_{t t}\right|}}, \quad \frac{d t}{d \lambda}= \pm \frac{\alpha}{g_{t t}},
$$

in whose derivation we have used Eq. (35).

With the previous choices, the integration over $\theta^{A}$ in Eq. (41) gives a factor of $V_{x}$, while we can employ Eq. (62) to perform a change of variables such that the integration over $\lambda$ is converted to be over $r$, resulting in

$$
S_{\text {null }}=\frac{2 \mathcal{N}}{\alpha} \int d r \sqrt{|g|} \Theta \log \left(l_{\text {null }} \Theta\right) .
$$

We can also use Eq. (62) to evaluate $\Theta$ while applying the chain rule, which gives 


$$
\Theta=\frac{\alpha}{\sqrt{|g|}} \partial_{r} \sqrt{\sigma}
$$

Substitution of this last expression further simplifies Eq. (63) into

$$
S_{\text {null }}=2 \mathcal{N} \int d r \log \left(\frac{\alpha l_{\text {null }}}{\sqrt{|g|}} \partial_{r} \sqrt{\sigma}\right) \partial_{r} \sqrt{\sigma}
$$

The crucial point extracted from the previous calculations is that the integrand is independent of $t_{0}$. For any $0 \leq t_{0} \leq t_{c}$ the integration over the four light sheets runs from $r_{\min }$ to $r_{\max }$,

$S_{\text {null }}\left(0 \leq t_{0} \leq t_{c}\right)=8 \mathcal{N} \int_{r_{\min }}^{r_{\max }} d r \log \left(\frac{\alpha l_{\text {null }}}{\sqrt{|g|}} \partial_{r} \sqrt{\sigma}\right) \partial_{r} \sqrt{\sigma}$

Hence, $S_{\text {null }}$ does not depend on $t_{0}$.

We thus conclude that $S_{\text {WDW }}$, and as a consequence the complexity of the state, is constant for $0 \leq t_{0} \leq t_{c}$.

\section{B. After the critical time: $t_{0}>t_{c}$}

As before, we start by evaluating the bulk contribution in Eq. (39) by using the definition of each region given in Eqs. (33) and (37). We have

$$
\begin{gathered}
S_{I}=2 \mathcal{N} \int_{r_{\text {min }}}^{r_{h}} d r \sqrt{-g} \mathcal{L}(r)\left(t_{0}-r_{\star}(r)\right), \\
S_{I I}=S_{I V}=-2 \mathcal{N} \int_{r_{h}}^{r_{\text {max }}} d r \sqrt{-g} \mathcal{L}(r) r_{\star}(r), \\
S_{I I I}=2 \mathcal{N} \int_{r_{m}}^{r_{h}} d r \sqrt{-g} \mathcal{L}(r)\left(-t_{0}-r_{\star}(r)\right) .
\end{gathered}
$$

Note that the $t_{0}$ dependence in $S_{I I I}$ is no longer canceled out by $S_{I}$. To see explicitly how this happens let us rewrite $S_{I I I}$ as

$$
\begin{aligned}
S_{I I I}= & -2 \mathcal{N} \int_{r_{\min }}^{r_{m}} d r \sqrt{-g} \mathcal{L}(r)\left(-t_{0}-r_{\star}(r)\right) \\
& +2 \mathcal{N} \int_{r_{\min }}^{r_{h}} d r \sqrt{-g} \mathcal{L}(r)\left(-t_{0}-r_{\star}(r)\right),
\end{aligned}
$$

where we use the fact that $r_{\min }<r_{m}$. Thus, using Eq. (45), the bulk action evaluated at the WDW patch for later times can be written as

$$
\begin{aligned}
S_{\text {bulk }}\left(t_{0}>t_{c}\right)= & S_{\text {bulk }}\left(0 \leq t_{0} \leq t_{c}\right) \\
& +2 \mathcal{N} \int_{r_{\text {mim }}}^{r_{m}} d r \sqrt{-g} \mathcal{L}(r)\left(t_{0}+r_{\star}(r)\right) .
\end{aligned}
$$

Note that $S_{\text {bulk }}\left(t_{0}>t_{c}\right)$ diverges as the regulator $r_{\max }$ is taken to infinity, but this behavior is contained in the $t_{0}$ independent term $S_{\text {bulk }}\left(t_{0}<t_{c}\right)$.

Next we turn to the surface integrals. Once again we set the contribution of the null hypersurfaces to zero by choosing an affine parameter. As the WDW patch no longer reaches the past singularity, we are left with

$$
S_{\text {surface }}=S_{\text {future }}+S_{\text {left }}+S_{\text {right }} .
$$

Using the previous expressions for the extrinsic curvature and induced metric on the hypersurfaces and the definition of the regions, we can compute the corresponding integrals. For each one we have

$$
\begin{aligned}
S_{\text {future }} & =-2 \mathcal{N} \mathcal{G}\left(r_{\text {min }}\right)\left(t_{0}-r_{\star}\left(r_{\text {min }}\right)\right), \\
S_{\text {right }} & =S_{\text {left }}=-2 \mathcal{N} \mathcal{G}\left(r_{\text {max }}\right) r_{\star}\left(r_{\text {max }}\right),
\end{aligned}
$$

and thus the total surface action is

$$
\begin{aligned}
S_{\text {surface }}\left(t_{0}>t_{c}\right)= & -4 \mathcal{N} \mathcal{G}\left(r_{\max }\right) r_{\star}\left(r_{\max }\right) \\
& -2 \mathcal{N G} \mathcal{G}\left(r_{\min }\right)\left(t_{0}-r_{\star}\left(r_{\min }\right)\right) .
\end{aligned}
$$

Note that the $t_{0}$ dependence coming from the integration at the future singularity regulator is no longer canceled out. Explicitly we have

$$
\begin{aligned}
S_{\text {surface }}\left(t_{0}>t_{c}\right)= & S_{\text {surface }}\left(0 \leq t_{0} \leq t_{c}\right) \\
& -2 \mathcal{N G} \mathcal{G}\left(r_{\min }\right)\left(t_{0}+r_{\star}\left(r_{\text {min }}\right)\right),
\end{aligned}
$$

where the $r_{\max } \rightarrow \infty$ divergence is contained in $S_{\text {surface }}\left(0 \leq t_{0} \leq t_{c}\right)$, which is independent of $t_{0}$.

Next we have the contribution coming from the joints. For later times we have only seven joints to consider, of which six are of the space null and time null type, as can be seen in Figs. 4(b) and 6(b). We can evaluate these using the one-forms and auxiliary vectors defined in the previous subsection. For the joints in region I we have

$$
S_{\text {joint }}=\left.2 \mathcal{N} \sqrt{\sigma} \log \left(\frac{\alpha}{\sqrt{\left|g_{t t}\right|}}\right)\right|_{r=r_{\min }},
$$

while for the joints in regions II and IV we have

$$
S_{\text {joint }}=-\left.2 \mathcal{N} \sqrt{\sigma} \log \left(\frac{\alpha}{\sqrt{\left|g_{t t}\right|}}\right)\right|_{r=r_{\max }} .
$$

In order to evaluate the contribution coming from the nullnull joint, we need to define the auxiliary vector appearing in Eq. (40). Following [54] we have 


$$
\hat{k_{1}}=\operatorname{sign}\left(g_{r r}\right) \sqrt{\left|\frac{g_{r r}}{g_{t t}}\right|} \partial_{r}-\partial_{t}
$$

which lives on the left past light sheet. Substitution of this into Eq. (40) gives

$$
S_{\text {null-null }}=\left.2 \mathcal{N} \sqrt{\sigma} \log \left(\frac{\alpha^{2}}{\left|g_{t t}\right|}\right)\right|_{r=r_{m}} .
$$

After adding all the joint contributions we have

$$
\begin{aligned}
S_{\text {joint }}\left(t_{0}>t_{c}\right)= & -\left.8 \mathcal{N} \sqrt{\sigma} \log \left(\frac{\alpha}{\sqrt{\left|g_{t t}\right|}}\right)\right|_{r=r_{\max }} \\
& +\left.4 \mathcal{N} \sqrt{\sigma} \log \left(\frac{\alpha}{\sqrt{\left|g_{t t}\right|}}\right)\right|_{r=r_{\text {min }}} \\
& +\left.2 \mathcal{N} \sqrt{\sigma} \log \left(\frac{\alpha^{2}}{\left|g_{t t}\right|}\right)\right|_{r=r_{m}} .
\end{aligned}
$$

Note that the $t_{0}$ dependence is implicit in $r_{m}$. To isolate this time dependence we can rewrite the previous expression as

$$
\begin{aligned}
S_{\text {joint }}\left(t_{0}>t_{c}\right)= & S_{\text {joint }}\left(0 \leq t_{0} \leq t_{c}\right) \\
& -\left.4 \mathcal{N} \sqrt{\sigma} \log \left(\frac{\alpha}{\sqrt{\left|g_{t t}\right|}}\right)\right|_{r=r_{\text {min }}} \\
& +\left.2 \mathcal{N} \sqrt{\sigma} \log \left(\frac{\alpha^{2}}{\left|g_{t t}\right|}\right)\right|_{r=r_{m}} .
\end{aligned}
$$

Finally, we have the contribution coming from the null counterterm. For later times the two past light sheets no longer reach the singularity and end at $r_{m}$ instead. Thus, we explicitly have

$$
\begin{aligned}
S_{\text {null }}\left(t_{0}>t_{c}\right)= & 4 \mathcal{N} \int_{r_{\min }}^{r_{\max }} d r \log \left(\frac{\alpha l_{\text {null }}}{\sqrt{|g|}} \partial_{r} \sqrt{\sigma}\right) \partial_{r} \sqrt{\sigma} \\
& +4 \mathcal{N} \int_{r_{m}}^{r_{\max }} d r \log \left(\frac{\alpha l_{\text {null }}}{\sqrt{|g|}} \partial_{r} \sqrt{\sigma}\right) \partial_{r} \sqrt{\sigma}
\end{aligned}
$$

In order to isolate the $t_{0}$ dependence of the last expression we rewrite the second term as an integral from $r_{\min }$ to $r_{\max }$ minus an integral from $r_{\min }$ to $r_{m}$, which gives

$$
\begin{aligned}
S_{\text {null }}\left(t_{0}>t_{c}\right)= & S_{\text {null }}\left(0 \leq t_{0} \leq t_{c}\right) \\
& -4 \mathcal{N} \int_{r_{\min }}^{r_{m}} d r \log \left(\frac{\alpha l_{\text {null }}}{\sqrt{|g|}} \partial_{r} \sqrt{\sigma}\right) \partial_{r} \sqrt{\sigma},
\end{aligned}
$$

where we have used Eq. (66).

We can now proceed to compute $d S_{\mathrm{WDW}} / d t_{0}$. By taking the derivative of Eq. (69) with respect to $t_{0}$ we obtain

$$
\frac{d S_{\text {bulk }}}{d t_{0}}=2 \mathcal{N} \int_{r_{\min }}^{r_{m}} d r \sqrt{-g} \mathcal{L}(r)
$$

where the contribution coming from the upper integration limit vanishes because of the equation that defines $r_{m}$ [Eq. (34)]. On the other hand, the derivative of the surface integral (73) gives

$$
\frac{d S_{\text {surface }}}{d t_{0}}=-2 \mathcal{N G}\left(r_{\min }\right)
$$

while the joint contribution is

$\frac{d S_{\text {joint }}}{d t_{0}}=\left.2 \mathcal{N} \sqrt{-\frac{g_{t t}}{g_{r r}}}\left(\sqrt{\sigma} \frac{g_{t t}^{\prime}}{g_{t t}}+\frac{\sigma^{\prime}}{2 \sqrt{\sigma}} \log \left(\frac{\alpha^{2}}{g_{t t}}\right)\right)\right|_{r=r_{m}}$,

where the prime denotes the derivative with respect to $r$ and we have used the expression for the derivative of $r_{m}$ with respect to $t_{0}$ [Eq. (35)]. We have also used the fact that $g_{t t}\left(r_{m}\right)>0$ and $g_{r r}\left(r_{m}\right)<0$. Finally, the $t_{0}$ derivative of the null counterterm (81) is

$$
\frac{d S_{\text {null }}}{d t_{0}}=-\left.4 \mathcal{N} \sqrt{-\frac{g_{t t}}{g_{r r}}} \frac{\sigma^{\prime}}{2 \sqrt{\sigma}} \log \left(\frac{\alpha l_{\text {null }}}{\sqrt{|g|}} \frac{\sigma^{\prime}}{2 \sqrt{\sigma}}\right)\right|_{r=r_{m}} .
$$

Putting it all together, we obtain the derivative of $S_{\mathrm{WDW}}$ with respect to $t_{0}$ for $t_{0}>t_{c}$ as

$$
\frac{d S_{\mathrm{WDW}}}{d t_{0}}=2 \mathcal{N}\left(\int_{r_{\min }}^{r_{m}} d r \sqrt{-g} \mathcal{L}(r)-\mathcal{G}\left(r_{\min }\right)+\left.\sqrt{-\frac{g_{t t}}{g_{r r}}}\left(\sqrt{\sigma} \frac{g_{t t}^{\prime}}{g_{t t}}-\frac{\sigma^{\prime}}{\sqrt{\sigma}} \log \left(\frac{\sigma^{\prime} l_{\text {null }}}{2 \sigma \sqrt{-g_{r r}}}\right)\right)\right|_{r=r_{m}}\right) .
$$

Note that, as it should, the addition of the null counterterm has eliminated the arbitrary normalization constant $\alpha$, but the dependence on the arbitrary length scale $l_{\text {null }}$ remains. However, as we will explicitly show numerically in the next section, this term vanishes in the limit $t_{0} / t_{c} \rightarrow \infty$ for all the geometries that we consider. We also present in
Appendix $\mathrm{B}$ an analytical proof of this vanishing that applies to metrics of the general form of Eq. (26) that we are considering. We would like to stress that, even if we used an affine parametrization in all of the previous calculations, the end result (86) would be invariant under reparametrizations of the null generators. 
Given that both the MT and DK families of solutions are not analytical, we need to evaluate this integral numerically. We will show the result of said evaluation below. It follows from Eq. (1) that the rate of change of the complexity of the TFD is related to $d S_{\mathrm{WDW}} / d t_{0}$ by

$$
\frac{d C}{d \tau}=\frac{1}{2} \frac{d S_{\mathrm{WDW}}}{d t_{0}}
$$

\section{Counterterms and the renormalization scheme}

From Eq. (86) we can see that all the terms that diverge in the $r_{\max } \rightarrow \infty$ limit were eliminated by the time derivative, rendering $d S_{\mathrm{WDW}} / d t_{0}$ finite. This is because the $r=r_{\max }$ surfaces only undergo a time translation and, given that both families of solutions are static, any boundary integral evaluated at this surface will be independent of $t_{0}$. This is true for the counterterm actions (11) and (21), which is why we did not include them in the evaluation of $S_{\text {WDW. }}$. It should be noted, though, that $S_{\mathrm{WDW}}$ itself is a divergent quantity. The counterterms necessary to remove said divergences were computed in [55] and later applied in [59] for a Bañados-Teitelboim-Zanelli (BTZ) black hole. These counterterms do not modify the late-time behavior of the complexity rate of change; thus, we omitted them from the previous computation.

Usually, when computing thermodynamic quantities such as the free energy of the state, the gravitational action is evaluated in the exterior region. The boundary of this region is constituted by the surfaces at $r=r_{\max }$ and $r=r_{h}$, but the counterterms vanish when evaluated at the horizon. However, as the WDW patch boundary includes the $r=$ $r_{\min }$ surfaces, the counterterms need to be evaluated there too. We explicitly checked numerically that, for any solution in either model, the contribution coming from this vanishes when the limit $r_{\min } \rightarrow r_{s}$ is taken. This means that $S_{\mathrm{WDW}}$, and as a consequence the complexity of the TFD state, is independent of the finite term determined by $C_{s c h}$.

\section{Results for the MT model}

For the case of the MT model we have, after using the equations of motion,

$$
\begin{gathered}
\mathcal{L}(r)=-8, \\
\mathcal{G}(r)=\frac{r^{4} e^{-\frac{5}{4} \phi}\left(r(\mathcal{F B})^{\prime}+\mathcal{B} \mathcal{F}\left(8-3 r \phi^{\prime}\right)\right)}{\sqrt{\mathcal{B}}}, \\
\sigma(r)=r^{6} e^{-\frac{5}{2} \phi} .
\end{gathered}
$$

With these expressions at hand, we can easily evaluate Eq. (86) numerically, which allows us to compute the rate of change of the complexity of the TFD state as a function of $\tau$

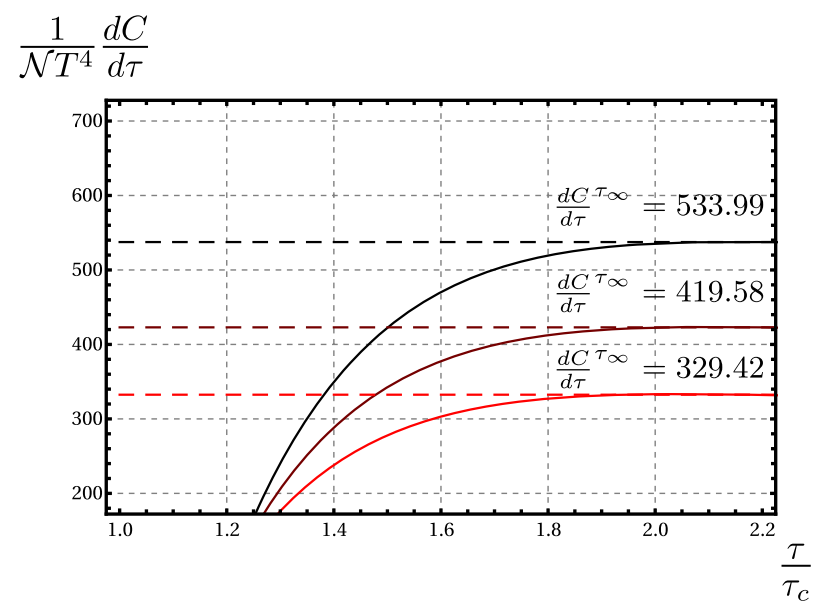

FIG. 7. Rate of change of the complexity $d C / d \tau$ in units of $\mathcal{N} T^{4}$ as a function of $\tau$ for the MT model. Each curve corresponds to a different value for the anisotropic parameter, that is, $a / T=$ $\{19.57,41.14,85.46\}$ from bottom to top. The horizontal dashed lines correspond to the late-time behavior of each curve, with the precise values being $\frac{d C}{d \tau} \tau_{\infty}=\{329.42,419.58,533.99\}$, respectively. For all cases we fixed $l_{\text {null }}=L=1$.

for any value of the anisotropic parameter. We show the result of said evaluation in Fig. 7 for $l_{\text {null }}=L=1$ and the three values of the anisotropy $a / T=\{19.57,41.14,85.46\}$, displayed from bottom to top. This shows that the general effect of the anisotropy is to increase the value of $d C / d \tau$ for any given $\tau$. We can also see that, for any $a / T$, at $\tau$ shortly after $\tau_{c}$ the rate of change of the complexity decreases as time passes, reaches a minimum, and then increases to a constant value, which we will denote as $\frac{d C}{d \tau} \tau_{\infty}$, for late times $\tau \gg \tau_{c}$. We have placed dashed horizontal lines in Fig. 7 to mark these asymptotic values and have plotted them as a continuous function of $a / T$ displayed as a red line in Fig. 8. While the early-time behavior can be modified by changing $l_{\text {null }}$, the

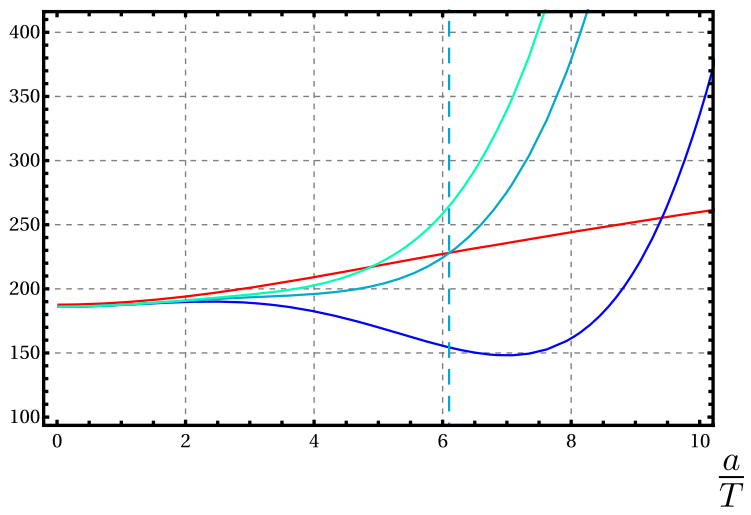

FIG. 8. Check of Lloyd's bound for the MT model as a function of $a / T$. The red curve (on the lower right side) corresponds to $\frac{d C}{d \tau} \tau_{\infty}$, while the curves in shades of blue correspond to the energy of the TFD state $2 E / \pi$ for $C_{s c h}=\{-2,-3,-5\}$ from left to right. All quantities are given in units of $\mathcal{N} T^{4}$. 


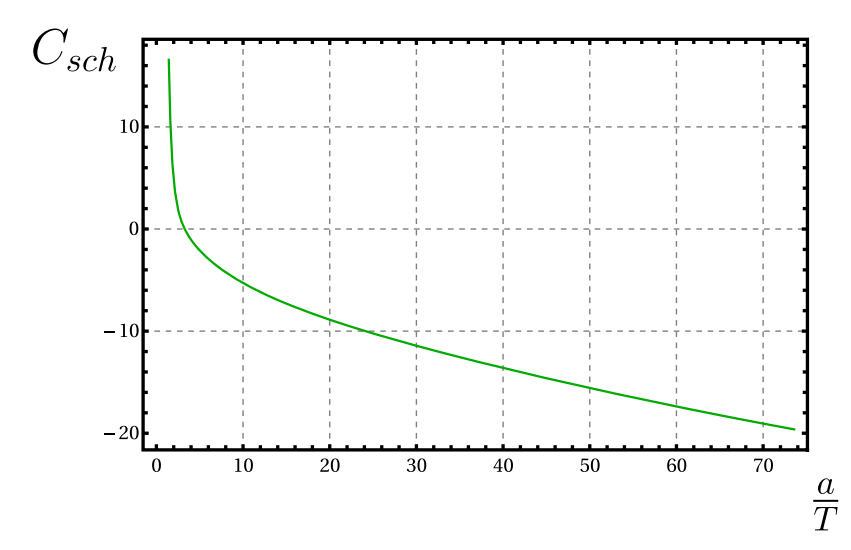

FIG. 9. Running of $C_{s c h}$ with $a / T$ to keep the renormalization condition of saturating Lloyd's bound in the MT model.

late-time behavior is independent of this arbitrary constant. It is also important to note that, for a given $\tau$, the rate of change of the complexity depends only on the dimensionless parameter $a / T$. We checked that this was the case numerically by varying $a$ and $T$ independently. We conclude from this that $d C / d \tau$ is independent of the energy scale $\mu$, and thus is unaffected by the conformal anomaly.

As mentioned above, in Fig. 8 we show the late-time behavior of the rate of change of the complexity $\frac{d C \tau_{\infty}}{d \tau}$ as a function of $a / T$ (red curve) and compare it to the energy of the state to see whether Lloyd's bound is verified at infinite time for the MT model, as this was the case for $a=0$. As explained in Sec. II, we have set the renormalization scale as $\mu=L=1$, but up to this point we still do not have a physical reason to fix the value of $C_{s c h}$. Thus, in Fig. 8 we plot the energy of the state as a function of $a / T$ for $C_{s c h}=$ $\{-2,-3,-5\}$ shown by the curves from left to right, in addition to the one representing $\frac{d C \tau_{\infty}}{d \tau}$. We see that at any value of $a / T$ Lloyd's bound at infinite time can be satisfied, saturated, or violated depending on the value of $C_{s c h}$, as we exemplify for $a / T=6.10$, shown by the vertical dashed line in Fig. 8. For this to be an actual bound regardless of how large the anisotropy is, it would be necessary to push $C_{s c h}$ all the way to $\infty$, invalidating all physical calculations. Instead, we suggest choosing the value of $C_{s c h}$ that, at each $a / T$, leads to the saturation of Lloyd's bound. We show this value of $C_{s c h}$ as a function of $a / T$ in Fig. 9. Physically, this amounts to using the saturation of Lloyd's bound at infinite time as a renormalization condition and letting $C_{s c h}$ run with $a / T$ to satisfy it.

\section{E. Results for the DK model}

For the case of the DK model we have, after using the equations of motion,

$$
\mathcal{L}(r)=-8-\frac{4 b^{2}}{3 V^{2}}
$$

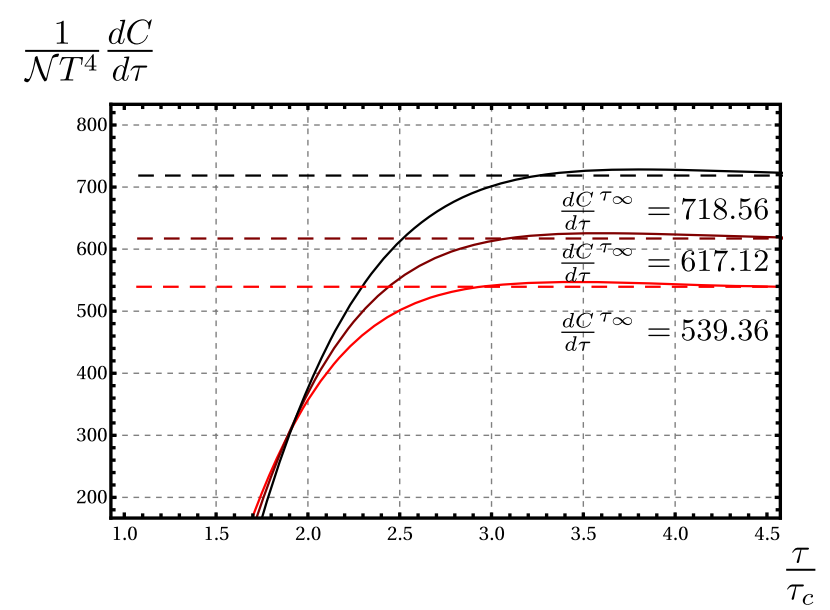

FIG. 10. Rate of change of the complexity $d C / d \tau$ in units of $\mathcal{N} T^{4}$ as a function of $\tau$ for the DK model. Each curve corresponds to a different magnetic field intensity, that is, $b / T^{2}=$ $\{40.59,47.56,56.62\}$ from bottom to top. The horizontal dashed lines correspond to the late-time behavior of each curve, with the precise values being $\frac{d C}{d \tau} \tau_{\infty}=\{539.36,617.12,718.56\}$ respectively. For all cases we fixed $l_{\text {null }}=L=1$.

$$
\begin{gathered}
\mathcal{G}(r)=\frac{U W V^{\prime}+(U W V)^{\prime}}{\sqrt{W}}, \\
\sigma(r)=W V^{2} .
\end{gathered}
$$

Employing these expressions we can evaluate Eq. (86) numerically, which allows us to compute the complexity rate of change on the TFD state as a function of $\tau$ for any magnetic field intensity. In Fig. 10 we show the result of this evaluation for $l_{\text {null }}=L=1$, with the three different values of the magnetic field intensity being, from bottom to top, $b / T^{2}=\{40.59,47.56,56.62\}$. We see that the magnetic field has the general effect of increasing the value of $d C / d \tau$ for any given $\tau$. The behavior common to the three plots in Fig. 10 indicates that for any $b / T^{2}$, at $\tau$ shortly past $\tau_{c}$ the complexity rate of change is an increasing function of time until it reaches a maximum, after which it becomes a decreasing function that for $\tau \gg \tau_{c}$ tends to a constant value, which we denote as $\frac{d C \tau_{\infty}}{d \tau}$ and indicate in the three cases of Fig. 10 as horizontal dashed lines. While the earlytime behavior can be modified by varying $l_{\text {null }}$, the latetime behavior is unaffected by it. We also explicitly checked to ensure that, for a given $\tau$, the rate of change of the complexity depends only on the dimensionless parameter $b / T^{2}$. In practice, we achieved this by varying $b$ and $T$ independently. We conclude from this that $d C / d \tau$ is independent of the energy scale $\mu$, proving that this quantity is unaffected by the conformal anomaly.

We show $\frac{d C}{d \tau} \tau_{\infty}$ as a function of $b / T^{2}$ (red curve) in Fig. 11 and compare it to the energy of the state in order to 


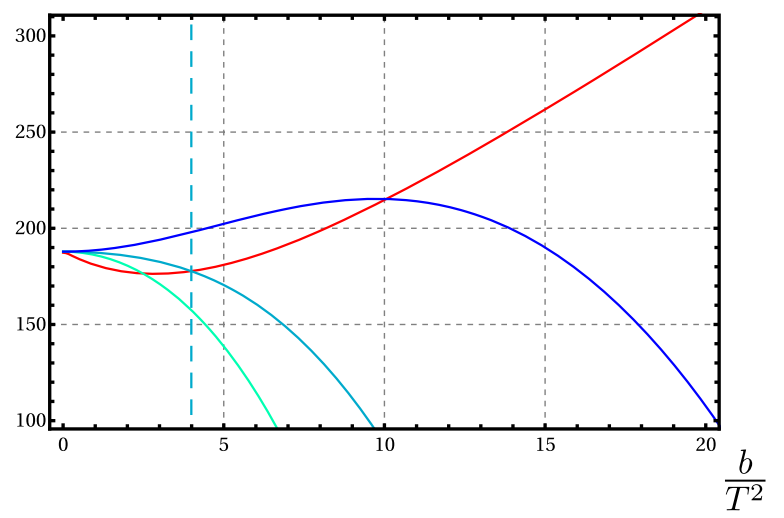

FIG. 11. Check of Lloyd's bound for the DK model as a function of $b / T^{2}$. The red curve (to the upper right) corresponds to $\frac{d C}{d \tau} \tau_{\infty}$, while the curves in shades of blue correspond to the energy of the TFD state $2 E / \pi$ for $C_{s c h}=\{0,1,2\}$ from left to right. All quantities are given in units of $\mathcal{N} T^{4}$.

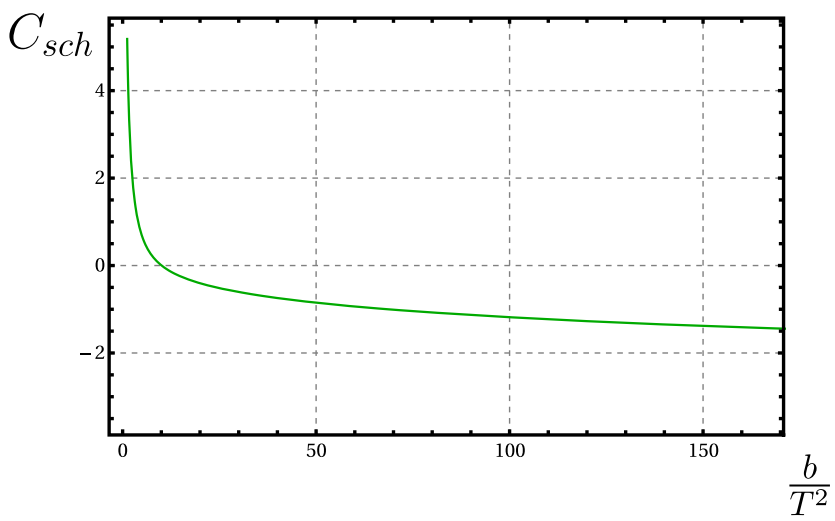

FIG. 12. Running of $C_{s c h}$ with $b / T^{2}$ to maintain the renormalization condition of the saturation of Lloyd's bound for the DK model.

check whether Lloyd's bound is verified in the DK model at infinite time, as was the case for $b=0$. We have fixed the renormalization scale $\mu=L=1$ but, as explained in Sec. II, up to this point of the analysis we do not have a physical reason to fix $C_{s c h}$. Hence, in Fig. 11 we show the plots of the energy of the state as a function of $b / T^{2}$ for $C_{s c h}=\{0,1,2\}$ (from left to right). As was the case for the MT model and as we exemplify at $b / T^{2}=3.99$, Lloyd's bound at infinite time can be satisfied, saturated, or violated for any $b / T^{2} \neq 0$ depending on the value of $C_{\text {sch }}$, and the only way to respect Lloyd's bound for any $b / T^{2}$ is by setting $C_{s c h}=\infty$. Once again we decide to adjust $C_{s c h}$ at each value of $b / T^{2}$ so that Lloyd's bound is saturated. As before, the latter corresponds to the physical proposal of letting $C_{s c h}$ run with $b / T^{2}$, as we show in Fig. 12, to maintain the renormalization condition of the saturation of Lloyd's bound at infinite time.

\section{DISCUSSION}

In this paper we employed holographic methods to determine the effect that the conformal anomaly has on the computational complexity. To this end, we extended the study presented in Eq. [31] to arbitrary anisotropies and magnetic field intensities by considering the numerical families of solutions for both the MT and DK models. While our results coincided with previous ones where applicable, we were able to derive many novel results.

Our first main result is that the rate of change of the complexity is unaffected by the conformal anomaly present in both models. The first of two reasons why this is the case is that $\left(1 / T^{4}\right) d C / d \tau$, which is a dimensionless quantity, depends only on $a$ and $T$ through the dimensionless ratio $a / T$, proving that such a derivative is independent of the energy scale $\mu$, which we fixed to unity throughout this paper. The second reason is that the counterterm action, which contains the scheme-dependent coefficient $C_{s c h}$, does not contribute to the derivative with respect to $\tau$, as the integral over the boundary regulator is constant and the integral near the singularity vanishes as the regulator is removed.

The other main result is in regard to Lloyd's bound. It is expected, when using the CA prescription, that Lloyd's bound will be violated for any finite time, only to be saturated when an infinite amount of time has passed. Knowing that this was the case for $a=0$ and $b=0$, we studied the validity of this result for arbitrary values of the anisotropic parameter and the magnetic field. As explained in Sec. I, the energy of the system, which appears on the right-hand side of Lloyd's bound (5), depends on both the energy scale $\mu$ and the coefficient $C_{s c h}$. However, as just stated, $d C / d \tau$, which appears on the right-hand side of Eq. (5), does not depend on any of these quantities. While at first sight this could be interpreted as an inconsistency for Lloyd's bound when a conformal anomaly is present, that is not what it is. The role of $C_{s c h}$ is to keep physical quantities scheme independent and, particularly for the energy, this means to absorb any modification that it could suffer when the value of $\mu$ is changed once, of course, a renormalization condition has been imposed. Thus, the above results demonstrate that, if so desired, the saturation of Lloyd's bound at infinite time can be used as a renormalization condition and lets $C_{s c h}$ be adjusted to satisfy it for any given $\{\mu, a, T\}$ or $\{\mu, b, T\}$, depending on the model.

We would like to stress the importance of $d C / d \tau$ being independent of both $\mu$ and $C_{s c h}$. If it had turned out to depend on only one of the two, there would have been no way to cancel any arbitrary change in the other, making it a scheme-dependent quantity, and therefore questioning the validity of the CA conjecture. Clearly the other acceptable option was for $d C / d \tau$ to depend on both $\mu$ and $C_{s c h}$, so we find it interesting to have disproved this possibility.

It is also important to note that, to our knowledge, a renormalization condition has not previously been proposed for either the MT or DK model. 


\section{ACKNOWLEDGMENTS}

We acknowledge partial financial support from PAPIITIN113618, UNAM. D. A., C. D., and Y. D. O. are supported by CONACYT Ph.D. grants. We would like to thank Gary Horowitz for sharing insight about the conformal anomaly in the holographic context.

\section{APPENDIX A: INTERIOR SOLUTIONS}

\section{MT MODEL}

In this Appendix we explain the integration procedure needed to obtain the interior solutions for the MT model. The equations of motion that determine the metric functions and the dilaton and axion fields come from the variation of the five-dimensional action (7). After the substitution of the Ansatz (8), these equations can be manipulated to write $\mathcal{F}$ and $\mathcal{B}$ in terms of $\phi$ as

$$
\begin{gathered}
\mathcal{F}=\frac{e^{-\frac{1}{2} \phi}\left(a^{2} e^{\frac{7}{2} \phi}\left(r \phi^{\prime}-4\right)+16 r^{3} \phi^{\prime}\right)}{4 r^{3}\left(\phi^{\prime}+r \phi^{\prime \prime}\right)}, \\
\frac{\mathcal{B}^{\prime}}{\mathcal{B}}=\frac{-16 \phi^{\prime}+9 r \phi^{\prime 2}-20 r \phi^{\prime \prime}}{24-10 r \phi^{\prime}},
\end{gathered}
$$

while the dilaton itself is given by the third order differential equation

$$
\begin{aligned}
0= & 8 r\left(r^{2}\left(11 a^{2} e^{\frac{7 \phi}{2}}+96 r^{2}\right) \phi^{\prime \prime 2}+12 a^{2} r e^{\frac{7 \phi}{2}} \phi^{\prime \prime \prime}\right)+8 r\left(12 a^{2} e^{\frac{7 \phi}{2}} \phi^{\prime \prime}\right)+\left(352 r^{5}-13 a^{2} r^{3} e^{\frac{7 \phi}{2}}\right) \phi^{\prime 4}+2 r \phi^{\prime 2}\left(r^{2}\left(23 a^{2} e^{\frac{7 \phi}{2}}-880 r^{2}\right) \phi^{\prime \prime}\right) \\
& +2 r \phi^{\prime 2}\left(5 r^{3} \phi^{\prime \prime \prime}\left(a^{2} e^{\frac{7 \phi}{2}}+16 r^{2}\right)-12 a^{2} e^{\frac{7 \phi}{2}}+960 r^{2}\right)+\phi^{\prime 3}\left(8 r^{2}\left(7 a^{2} e^{\frac{7 \phi}{2}}-200 r^{2}\right)\right)+\phi^{\prime 3}\left(\left(352 r^{6}-13 a^{2} r^{4} e^{\frac{7 \phi}{2}}\right) \phi^{\prime \prime}\right) \\
& -2 \phi^{\prime}\left(32 r^{2}\left(a^{2} e^{\frac{7 \phi}{2}}-30 r^{2}\right) \phi^{\prime \prime}+15 r^{4}\left(a^{2} e^{\frac{7 \phi}{2}}+16 r^{2}\right) \phi^{\prime \prime 2}+32 r^{3} \phi^{\prime \prime \prime}\left(a^{2} e^{\frac{7 \phi}{2}}+6 r^{2}\right)\right)+15 r^{4}\left(48 a^{2} e^{\frac{7 \phi}{2}}\right) .
\end{aligned}
$$

As explained in [33], it is possible to eliminate the anisotropic parameter $a$ from Eq. (A3) altogether by shifting the dilaton field to

$$
\tilde{\phi}=\phi+\frac{4}{7} \log a,
$$

and thus solve directly for $\tilde{\phi}$. This allows us to easily impose the boundary condition $\phi_{b d r y}=0$ by defining that the anisotropy corresponding to a given solution is given by

$$
a=e^{\frac{7}{4} \tilde{\phi}_{b d r y} .}
$$

Thus, solutions with different $\tilde{\phi}_{b d r y}$ correspond to solutions with different anisotropies.

Given that the equation for $\tilde{\phi}$ is highly nonlinear, it is necessary to resort to numerical methods to obtain a solution for an arbitrary $a / T$. The first step is to expand Eq. (A3) in a power series of $r$ around the horizon

$$
\tilde{\phi}=\tilde{\phi}_{h}+\sum_{i=1}^{\infty} \tilde{\phi}_{i}\left(r-r_{h}\right)^{i}
$$

By solving order by order it is possible to write any undetermined coefficient $\phi_{i}$ in terms of $\tilde{\phi}_{h}$ [60]. Then Eq. (A6) is used to provide initial data for the numerical integration, starting from $r=r_{h}+\epsilon$, with $\epsilon \ll r_{h}$, all the way to the boundary at $r=\infty$ in the case of the exterior solutions, and from $r=r_{h}-\epsilon$ toward, and down to, the singularity at $r=0$ in the case of the interior solutions. After this we can obtain the anisotropy by substitution into Eq. (A5) and finally $\phi$ with the appropriate boundary condition using Eq. (A4).
With the solution for $\phi$ in the interior and the exterior at hand, we can obtain $\mathcal{F}$ by simply substituting into Eq. (A1), as this is an algebraic relation. Incidentally, this always results in an $\mathcal{F}$ satisfying the boundary condition $\mathcal{F}_{\text {bdry }}=1$. However, given that the relation between $\mathcal{B}$ and $\phi$ is differential, it is necessary to again perform a numerical integration. In order to do this we expand Eq. (A2) in a power series of $r$ around $r_{h}$ using Eq. (A6) and

$$
\mathcal{B}=\mathcal{B}_{h}+\sum_{i=1}^{\infty} \mathcal{B}_{i}\left(r-r_{h}\right)^{i}
$$

By solving Eq. (A2) order by order, we can write any undetermined coefficient in terms of $\mathcal{B}_{h}$ and $\phi_{h}$. Then, using the result of this as our initial data, we can integrate Eq. (A2) numerically from $r=r_{h}+\epsilon$ to the boundary at $r=\infty$ for the exterior solutions, and from $r=r_{h}-\epsilon$ to the singularity at $r=0$ for the interior solutions. Given that we want the spacetime to asymptote $\mathrm{AdS}_{5}$ at the boundary, the last step is to use the symmetry of Eq. (A2) and redefine

$$
\mathcal{B} \rightarrow \frac{\mathcal{B}}{\mathcal{B}_{b d r y}}
$$

ensuring in this way that $\mathcal{B}$ goes to unity at the boundary. Note that this rescaling needs to be done consistently for both the exterior and interior solutions. In Fig. 13 we show the metric functions for small $(a / T=4.41)$ and large $(a / T=24.91)$ anisotropies in the interior and exterior regions. 


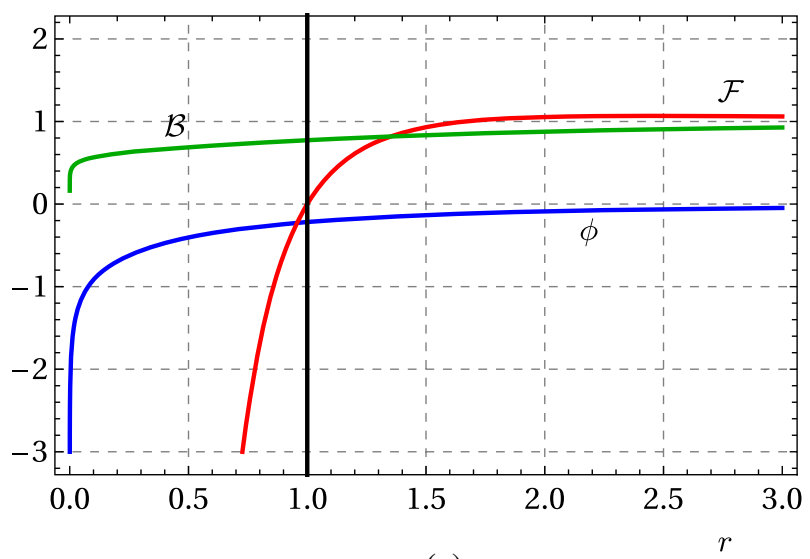

(a)

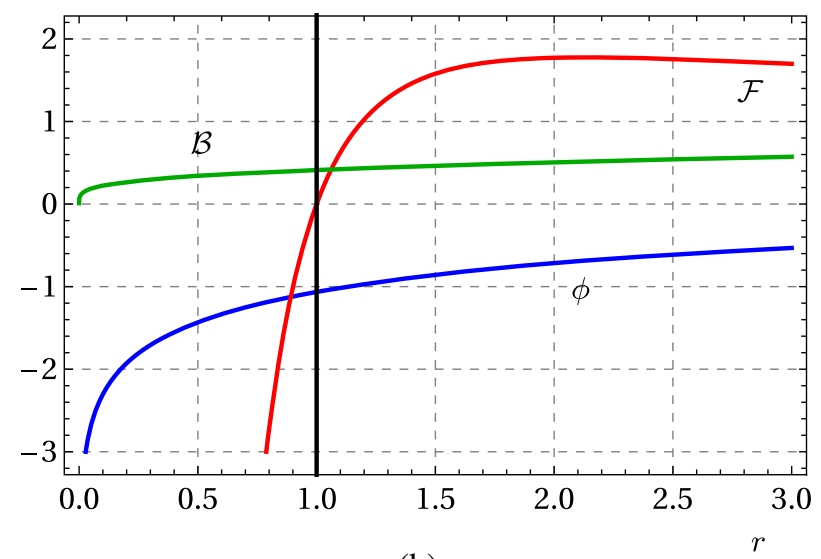

(b)

FIG. 13. Metric functions and dilaton for the MT model as functions of $r$ for (a) $a / T=4.41$ and (b) $a / T=24.91$. In each panel the horizon, located at $r_{h}=1$, is denoted as a black vertical line.

\section{DK MODEL}

In this section we show the integration procedure needed to obtain the interior solutions for the DK model. The equations of motion that determine the metric functions and the Maxwell field come from the variation of the fivedimensional action (17). After the substitution of the Ansatz (18), Maxwell equations are automatically satisfied and Einstein equations can be manipulated into

$$
\begin{aligned}
0= & 2 W^{2}\left(4 b^{2}+V\left(U^{\prime} V^{\prime}+U V^{\prime \prime}\right)\right) \\
& -V W\left(2 V\left(U^{\prime} W^{\prime}+U W^{\prime \prime}\right)+U V^{\prime} W^{\prime}\right)+U V^{2} W^{\prime 2}, \\
0= & -2 W^{2}\left(V^{\prime 2}-2 V V^{\prime \prime}\right)+2 V^{2} W W^{\prime \prime}-V^{2} W^{\prime 2}, \\
0= & W\left(8 b^{2}-6 V^{2}\left(U^{\prime \prime}-8\right)-6 V U^{\prime} V^{\prime}\right)-3 V^{2} U^{\prime} W^{\prime}, \\
0= & W\left(4 b^{2}+2 V U^{\prime} V^{\prime}+U V^{\prime 2}-24 V^{2}\right) \\
& +V W^{\prime}\left(V U^{\prime}+2 U V^{\prime}\right) .
\end{aligned}
$$

Given that the equations are singular at $r_{h}$, in order to solve numerically we first expand them in powers of $r$ around $r_{h}$ by using

$$
\begin{aligned}
& U=6 r_{h}\left(r-r_{h}\right)+\sum_{i=2}^{\infty} U_{i}\left(r-r_{h}\right)^{i}, \\
& V=V_{0}+\sum_{i=1}^{\infty} V_{i}\left(r-r_{h}\right)^{i}, \\
& W=3 r_{h}^{2}+\sum_{i=1}^{\infty} W_{i}\left(r-r_{h}\right)^{i} .
\end{aligned}
$$

This behavior near the horizon allows the family of solutions to easily interpolate between the black D3-brane and $\mathrm{BTZ} \times \mathbb{R}^{2}$ by changing the value of $b / V_{0}$ from 0 to $\sqrt{3}$. Additionally, this also ensures that the temperature of every member of the family is given by $T=3 r_{h} / 2 \pi$.
However, it is important to note that the coordinate $r$ here is not the usual radial coordinate of the black brane solution $\tilde{r}$. Instead, the relation between the two is given by

$$
\tilde{r}=r+\frac{r_{h}}{2},
$$

which means that for a vanishing magnetic field the singularity is located at $r=-r_{h} / 2$, not at $r=0$.

By plugging Eq. (A10) into Eq. (A9) we can solve for any of the undetermined coefficients in terms of $b / V_{0}$ and then use this to provide initial data for the numerical integration. This is performed from $r=r_{h}+\epsilon$ to the boundary at $r=\infty$ for the exterior solutions, and from $r=$ $r_{h}-\epsilon$ to the singularity at $r=r_{s}$ for the interior solutions, with $\epsilon \ll r_{h}$ in both cases. This procedure will give solutions whose boundary behavior is

$$
V \sim V_{b d r y} r^{2}, \quad W \sim W_{b d r y} r^{2}, \quad U \sim r^{2} .
$$

However, we can exploit the symmetries of the equations of motion (A9) and rescale them as

$$
V \rightarrow \frac{V}{V_{b d r y}}, \quad W \rightarrow \frac{W}{W_{b d r y}}, \quad b \rightarrow \frac{b}{V_{b d r y}},
$$

which in turn gives the desired $\mathrm{AdS}_{5}$ behavior at the boundary. Once again this rescaling needs to be done consistently for both the exterior and interior solutions. Notice that on this occasion it is necessary to simultaneously scale the value of $b$ to preserve the solution.

One important thing to note is that the position of the singularity is fixed at $r_{s}=-r_{h} / 2$ not for every member of the family of solutions but only for $b / T^{2}=0$. Instead, the location of the singularity in the $r$ coordinate is a function of the magnetic field intensity. By $r_{s}$ we mean the radius at which the curvature scalar 


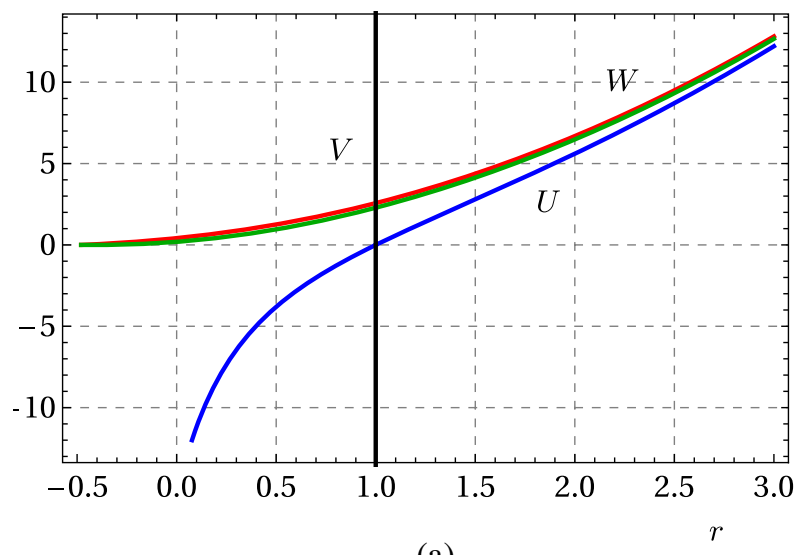

(a)

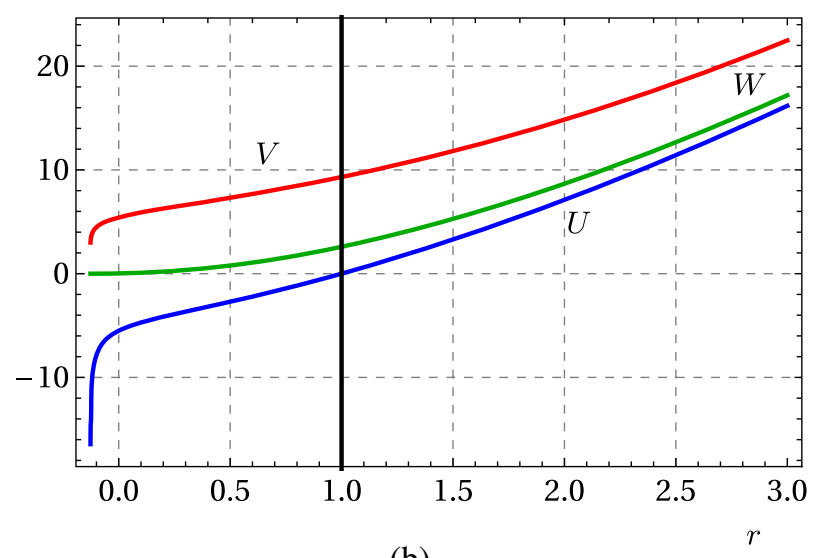

(b)

FIG. 14. Metric functions for the DK model as functions of $r$ for (a) $b / T^{2}=5.85$ and (b) $b / T^{2}=56.62$. In both panels the horizon, located at $r_{h}=1$, is denoted as a black vertical line.

$$
R_{\mu \nu \alpha \beta} R^{\mu \nu \alpha \beta}
$$

goes to infinity. We show the metric functions for small $\left(b / T^{2}=5.85\right)$ and large $\left(b / T^{2}=56.62\right)$ magnetic field intensities in the interior and exterior regions in Fig. 14.

\section{APPENDIX B: LATE-TIME BEHAVIOR OF THE COMPLEXITY RATE OF CHANGE}

In this section we explicitly write the expression for the late-time behavior of the complexity rate of change (86) and show that the dependence on $l_{\text {null }}$ vanishes in the limit $t_{0} \rightarrow \infty$. In this limit $r_{m}$ goes to $r_{h}$; hence, Eq. (86) takes the form

$$
\lim _{t_{0} \rightarrow \infty} \frac{d S_{\mathrm{WDW}}}{d t_{0}}=2 \mathcal{N}\left(\int_{r_{\min }}^{r_{h}} d r \sqrt{-g} \mathcal{L}(r)-\mathcal{G}\left(r_{\min }\right)\right)+2 \mathcal{N} \lim _{r_{m} \rightarrow r_{h}}\left(\left.\sqrt{-\frac{g_{t t}}{g_{r r}}}\left(\sqrt{\sigma} \frac{g_{t t}^{\prime}}{g_{t t}}-\frac{\sigma^{\prime}}{\sqrt{\sigma}} \log \left(\frac{\sigma^{\prime} l_{\text {null }}}{2 \sigma \sqrt{-g_{r r}}}\right)\right)\right|_{r=r_{m}}\right) .
$$

To evaluate the limit in the second term we use the fact that $g_{t t}$ has a zero at $r_{h}, g_{r r}$ has a simple pole there, and the other metric functions are regular at the horizon. This means that to leading order

$$
\sqrt{-\frac{g_{t t}}{g_{r r}}} \sim r-r_{h}, \quad \frac{\sigma^{\prime}}{\sqrt{\sigma}} \sim\left(r-r_{h}\right)^{0}, \quad \frac{\sigma^{\prime}}{\sigma} \sim\left(r-r_{h}\right)^{0} .
$$

Hence,

$$
\log \left(\frac{\sigma^{\prime} l_{\text {null }}}{2 \sigma \sqrt{-g_{r r}}}\right) \sim \log \left(r-r_{h}\right)
$$

which in turn means that

$$
\lim _{r_{m} \rightarrow r_{h}} \sqrt{-\frac{g_{t t}}{g_{r r}}} \frac{\sigma^{\prime}}{\sqrt{\sigma}} \log \left(\frac{\sigma^{\prime} l_{\text {null }}}{2 \sigma \sqrt{-g_{r r}}}\right)=0
$$

This shows that $l_{\text {null }}$ does not affect the late-time behavior of the rate of change of the complexity, as the final expression is

$$
\lim _{t_{0} \rightarrow \infty} \frac{d S_{\mathrm{WDW}}}{d t_{0}}=2 \mathcal{N}\left(\int_{r_{\min }}^{r_{h}} d r \sqrt{-g} \mathcal{L}(r)-\mathcal{G}\left(r_{\min }\right)+\left.\left(g_{t t}^{\prime} \sqrt{-\frac{\sigma}{g_{r r} g_{t t}}}\right)\right|_{r=r_{h}}\right) .
$$


[1] J. M. Maldacena, The large $N$ limit of superconformal field theories and supergravity, Int. J. Theor. Phys. 38, 1113 (1999); Adv. Theor. Math. Phys. 2, 231 (1998).

[2] S. S. Gubser, I. R. Klebanov, and A. M. Polyakov, Gauge theory correlators from noncritical string theory, Phys. Lett. B 428, 105 (1998).

[3] E. Witten, Anti-de Sitter space and holography, Adv. Theor. Math. Phys. 2, 253 (1998).

[4] S. Ryu and T. Takayanagi, Holographic Derivation of Entanglement Entropy from AdS/CFT, Phys. Rev. Lett. 96, 181602 (2006).

[5] V.E. Hubeny, M. Rangamani, and T. Takayanagi, A covariant holographic entanglement entropy proposal, J. High Energy Phys. 07 (2007) 062.

[6] T. Takayanagi and K. Umemoto, Entanglement of purification through holographic duality, Nat. Phys. 14, 573 (2018).

[7] G. Penington, Entanglement wedge reconstruction and the information paradox, J. High Energy Phys. 09 (2020) 002.

[8] A. Almheiri, R. Mahajan, J. Maldacena, and Y. Zhao, The page curve of Hawking radiation from semiclassical geometry, J. High Energy Phys. 03 (2020) 149.

[9] A. Almheiri, R. Mahajan, and J. Maldacena, Islands outside the horizon, arXiv:1910.11077.

[10] S. A. Hartnoll, G. T. Horowitz, J. Kruthoff, and J. E. Santos, Diving into a holographic superconductor, SciPost Phys. 10, 009 (2021).

[11] T. Hartman and J. Maldacena, Time evolution of entanglement entropy from black hole interiors, J. High Energy Phys. 05 (2013) 014.

[12] L. Susskind, Computational complexity and black hole horizons, Fortschr. Phys. 64, 24 (2016); 64, 44(A) (2016).

[13] D. Stanford and L. Susskind, Complexity and shock wave geometries, Phys. Rev. D 90, 126007 (2014).

[14] A. R. Brown, D. A. Roberts, L. Susskind, B. Swingle, and Y. Zhao, Holographic Complexity Equals Bulk Action?, Phys. Rev. Lett. 116, 191301 (2016).

[15] A. R. Brown, D. A. Roberts, L. Susskind, B. Swingle, and Y. Zhao, Complexity, action, and black holes, Phys. Rev. D 93, 086006 (2016).

[16] M. Alishahiha, Holographic complexity, Phys. Rev. D 92, 126009 (2015).

[17] S. Lloyd, Ultimate physical limits to computation, Nature (London) 406, 1047 (2000).

[18] L. Lehner, R. C. Myers, E. Poisson, and R. D. Sorkin, Gravitational action with null boundaries, Phys. Rev. D 94, 084046 (2016).

[19] D. Carmi, S. Chapman, H. Marrochio, R. C. Myers, and S. Sugishita, On the time dependence of holographic complexity, J. High Energy Phys. 11 (2017) 188.

[20] W. Cottrell and M. Montero, Complexity is simple!, J. High Energy Phys. 02 (2018) 039.

[21] J. Couch, S. Eccles, W. Fischler, and M.-L. Xiao, Holographic complexity and noncommutative gauge theory, J. High Energy Phys. 03 (2018) 108.

[22] B. Swingle and Y. Wang, Holographic complexity of Einstein-Maxwell-Dilaton gravity, J. High Energy Phys. 09 (2018) 106.

[23] M. Alishahiha, A. F. Astaneh, M. R. M. Mozaffar, and A. Mollabashi, Complexity growth with Lifshitz scaling and hyperscaling violation, J. High Energy Phys. 07 (2018) 042.
[24] M. Alishahiha, A. F. Astaneh, A. Naseh, and M. H. Vahidinia, On complexity for $F(R)$ and critical gravity, J. High Energy Phys. 05 (2017) 009.

[25] S. Mahapatra and P. Roy, On the time dependence of holographic complexity in a dynamical Einstein-dilaton model, J. High Energy Phys. 11 (2018) 138.

[26] D. Chen and J. Jiang, Investigating the complexity-equalsaction conjecture in regular magnetic black holes, Classical Quantum Gravity 37, 135003 (2020).

[27] H. Babaei-Aghbolagh, K. B. Velni, D. M. Yekta, and H. Mohammadzadeh, Holographic complexity for black branes with momentum relaxation, arXiv:2009.01340.

[28] R.-Q. Yang, Yu.-S. An, C. Niu, C.-Y. Zhang, and K.-Y. Kim, What kind of "complexity" is dual to holographic complexity?, arXiv:2011.14636.

[29] M. A. Nielsen, M. R. Dowling, M. Gu, and A. C. Doherty, Quantum computation as geometry, Science 311, 1133 (2006).

[30] M. Doroudiani, A. Naseh, and R. Pirmoradian, Complexity for charged thermofield double states, J. High Energy Phys. 01 (2020) 120.

[31] S. A. H. Mansoori, V. Jahnke, M. M. Qaemmaqami, and Y. D. Olivas, Holographic complexity of anisotropic black branes, Phys. Rev. D 100, 046014 (2019).

[32] D. Mateos and D. Trancanelli, Anisotropic $\mathcal{N}=4$ SuperYang-Mills Plasma and Its Instabilities, Phys. Rev. Lett. 107, 101601 (2011).

[33] D. Mateos and D. Trancanelli, Thermodynamics and instabilities of a strongly coupled anisotropic plasma, J. High Energy Phys. 07 (2011) 054.

[34] E. D'Hoker and P. Kraus, Magnetic brane solutions in AdS, J. High Energy Phys. 10 (2009) 088.

[35] M. Henningson and K. Skenderis, The holographic Weyl anomaly, J. High Energy Phys. 07 (1998) 023.

[36] S. de Haro, S. N. Solodukhin, and K. Skenderis, Holographic reconstruction of space-time and renormalization in the AdS/CFT correspondence, Commun. Math. Phys. 217, 595 (2001).

[37] I. Papadimitriou and K. Skenderis, Thermodynamics of asymptotically locally AdS spacetimes, J. High Energy Phys. 08 (2005) 004.

[38] K. Skenderis, Lecture notes on holographic renormalization, Classical Quantum Gravity 19, 5849 (2002).

[39] M. Bianchi, D. Z. Freedman, and K. Skenderis, Holographic renormalization, Nucl. Phys. B631, 159 (2002).

[40] M. Bianchi, D. Z. Freedman, and K. Skenderis, How to go with an RG flow, J. High Energy Phys. 08 (2001) 041.

[41] R. P. Martinez-y-Romero, L. Patino, and T. RamirezUrrutia, Increase of the energy necessary to probe ultraviolet theories due to the presence of a strong magnetic field, J. High Energy Phys. 11 (2017) 104.

[42] Our curvature convention for both models is $R_{\beta \mu \nu}^{\alpha}=$ $\partial_{\mu} \Gamma_{\beta \nu}^{\alpha}+\Gamma_{\mu \sigma}^{\alpha} \Gamma_{\beta \nu}^{\sigma}-(\mu \leftrightarrow \nu)$ and $R_{\mu \nu}=R_{\mu \sigma \nu}^{\sigma}$.

[43] Taking $L=1$ implies that $G_{5}=\pi / 2 N_{c}^{2}$, with $N_{c}$ the number of color degrees of freedom in the dual theory.

[44] C. Ecker, C. Hoyos, N. Jokela, D. R. Fernández, and A. Vuorinen, Stiff phases in strongly coupled gauge theories with holographic duals, J. High Energy Phys. 11 (2017) 031.

[45] M. Cvetic, M. J. Duff, P. Hoxha, J. T. Liu, H. Lu, J. X. Lu, R. Martinez-Acosta, C. N. Pope, H. Sati, and T. A. Tran, 
Embedding AdS black holes in ten and eleven dimensions, Nucl. Phys. B558, 96 (1999).

[46] U. Elinos and L. Patiño, Fundamental Landau levels in a strongly couple plasma, arXiv:2104.07716.

[47] D. Ávila and L. Patiño, Melting holographic mesons by cooling a magnetized quark gluon plasma, J. High Energy Phys. 06 (2020) 010.

[48] D. Areán, L. A. P. Zayas, L. Patiño, and M. Villasante, Velocity statistics in holographic fluids: Magnetized quarkgluon plasma and superfluid flow, J. High Energy Phys. 10 (2016) 158.

[49] D. Avila, V. Jahnke, and L. Patiño, Chaos, diffusivity, and spreading of entanglement in magnetic branes, and the strengthening of the internal interaction, J. High Energy Phys. 09 (2018) 131.

[50] J. F. Fuini and L. G. Yaffe, Far-from-equilibrium dynamics of a strongly coupled non-Abelian plasma with non-zero charge density or external magnetic field, J. High Energy Phys. 07 (2015) 116.

[51] G. Endrodi, M. Kaminski, A. Schafer, J. Wu, and L. Yaffe, Universal magnetoresponse in QCD and $\mathcal{N}=4$ SYM, J. High Energy Phys. 09 (2018) 070.

[52] Of course, the MT and DK models satisfy these conditions for any anisotropy and magnetic field.
[53] Note that this means that $\tau=2 t_{0}$, with $\tau$ the time variable relevant for the evolution of the TFD state mentioned in the Introduction.

[54] D. Carmi, R. C. Myers, and P. Rath, Comments on holographic complexity, J. High Energy Phys. 03 (2017) 118.

[55] A. Akhavan and F. Omidi, On the role of counterterms in holographic complexity, J. High Energy Phys. 11 (2019) 054.

[56] S. Chapman, H. Marrochio, and R. C. Myers, Complexity of formation in holography, J. High Energy Phys. 01 (2017) 062.

[57] This term is crucial when the spacetime under consideration is not stationary, as in the case of Vaidya spacetime [S. Chapman, H. Marrochio, and R. C. Myers, J. High Energy Phys. 06 (2018) 046; 06 (2018) 114].

[58] A. Reynolds and S. F. Ross, Divergences in holographic complexity, Classical Quantum Gravity 34, 105004 (2017).

[59] F. Omidi, Regularizations of action-complexity for a pure BTZ black hole microstate, J. High Energy Phys. 07 (2020) 020 .

[60] It is also necessary to impose the condition that $\mathcal{F}\left(r_{h}\right)=0$ by means of Eq. (A1). 